\title{
A Novel Fast Model Predictive Control with Actuator Saturation for \\ Large-Scale Structures
}

\author{
Haijun Peng*, Fei Li, Sheng Zhang and Biaosong Chen \\ Department of Engineering Mechanics, State Key Laboratory of Structural Analysis of Industrial \\ Equipment, Dalian University of Technology, Dalian 116024, China
}

\begin{abstract}
One of the most critical issues faced in the application of active control to engineering structures is actuator saturation. In this paper, a novel fast model predictive control with actuator saturation for large-scale structures is proposed. First, based on the second-order dynamic equation, the explicit expression form of the Newmark- $\beta$ method is derived. Then, according to the parametric variational principle, the explicit structure of the model predictive control (MPC) saturation controller is obtained. A linear complementary problem for the proposed fast MPC saturation controller is developed, replacing the quadratic programming problem for the original MPC saturation controller. The optimal control input can be achieved by solving one linear complementarity problem and one transient analysis problem. Particularly, the physical meaning of the explicit expression form of the Newmark- $\beta$ method is discovered and applied for increasing computational efficiency and saving memory. Finally, numerical simulations of a plane adjacent frame building subjected to earthquake ground motion demonstrate that the proposed fast MPC saturation controller is highly efficient and can be applied under a large step-length, especially for large-scale structural dynamic control problems.
\end{abstract}

\section{Keywords}

Large-scale structures; Actuator saturation; Fast model predictive control; Explicit expression form; Quadratic programming; Linear complementary

*Corresponding author. Email address: hjpeng@ dlut.edu.cn (H. J. Peng). 


\section{Introduction}

Structural active control is one of the most important research fields in civil engineering [1-5], particularly with relevant applications within the wind engineering and seismic engineering fields. Although passive control and/or semi-active control do not have a large power demand and offer high reliability at the expense of reducing control effectiveness, active control has still been a hot topic in recent years. In the past several years, great progress has been made in advancing the theory and practice of structural active control methods, such as classic control methods, including PID control [6], positive position feedback (PPF) [7], and velocity feedback control (VFC) [8], and modern control methods, including linear quadratic optimal control [9,10], Hळ theories [11,12], sliding mode control [13, 14], and neural networks $[15,16]$, which were developed to reduce building vibration under earthquake or wind excitation. However, when designing a controller for the active vibration control of structures, besides the control performance that must be considered, some practical issues should be considered in the controller design process as well [17].

In practical cases, actuator saturation [18, 19], unavoidable input time delay [20, 21] and parameter uncertainties of controlled structures [22,23] can cause a controller designed for structures without considering these factors to lose stability and even fail to work [24]. In this paper, the case of actuator saturation is one of most important factors and will be paid more attention. Saturation of the control input often occurs because the expected control input is unrealistic. Physical inputs such as force and torque are ultimately limited, and unexpected large amplitude disturbances can also push a system's actuators into saturation [25]. Therefore, control actuators are usually designed and implemented in such a way to operate below their physical limits. However, this approach is not acceptable from an economic viewpoint and can become unsafe under extreme loading conditions. A more rigorous approach should directly consider the system's limitations in the control algorithm [26]. Control actuators designed based on system limitations can be roughly classified into two categories: those that attempt to a priori prevent the system from reaching its limits and those that allow the system to reach its limits in a controlled manner [19]. For the former set of methods, a set of variable feedback gains is designed as a function of a single variable that indicates a trade-off between the reduction of the building response and the amplitude of the auxiliary mass stroke [27]. The on-off nonlinear velocity feedback control, as the natural evolution of the linear velocity feedback control, is employed when high gains and/or significant 
vibration levels are present together with saturation in the control law [28]. For the latter methods, control functions are considered to be piecewise constants and switching points are taken as decision variables, and then the bang-bang control problem is converted into a mathematical programming problem [29]. The model predictive control (MPC) is employed and transforms the control saturation problem into a parameter optimization problem [30]. The advantage of MPC is that the control saturation can be directly considered and designed in a simple manner.

With the rapid development of computer technology, MPC is becoming a reality in many engineering applications [31], such as civil engineering [32], mechanical engineering [33] and aerospace engineering [34]. The primary advantage of MPC over other control strategies is that it can handle process constraints that arise from natural requirements on an algorithmic level [35]. Despite the success of MPC in many engineering applications, there is still much room for improvement. For example, the time required to compute the control law in MPC cannot exceed the sampling time of the controller. In other words, the time necessary to evaluate the control law must be smaller than the sampling period [2]. Thus, multi-parametric explicit MPC $[36,37]$ methods transfer much online computational work into offline operations. The main drawback of this method is the complexity of the controller, with its increased dimensions and long horizons. The Newton-Raphson-based MPC [38, 39] methods proposed as alternate formulations of the MPC problem usually imply a loss of optimality.

From the above references, we can see that although MPC has shown to be effective in accounting for physical constraints and can provide satisfactory control performance, the application of MPC to the large-scale structure actuator saturation problem requires to carry out expensive computations in real time. To obtain a MPC controller including physical limits, the MPC saturation controller is newly proposed in this paper for satisfying the requirement of real-time large-scale computation. The proposed saturation controller is designed and constructed based on the two main aspects. One factor is an explicit expression form of discrete recursive dynamic equation for large-scale structures. Another factor is a linear complementarity problem obtained by the parametric variational principle. In the next section, two kinds of discretization scheme of the standard MPC (i.e. MPC1 and MPC2) will be presented for comparisons. The main contributions of this paper lie in two factors. First, the explicit structure of the MPC saturation controller can be obtained by the parametric variational principle. Meanwhile, the explicit MPC saturation controller is made up of one transient response analysis problem and one linear 
complementary problem. However, the original standard MPC1 and MPC2 saturation controller entirely rely on the on-line numerical solutions of parameter optimization. Second, based on the explicit expression form of the Newmark- $\beta$ method, the optimal control input can be derived from the second-order dynamic equation without forming an expanded state-space equation. The essential advantages of employing explicit expression form are reflected at the high efficiency and low memory requirements. Specifically, the computation of the matrix exponential is avoided and replaced by two off-line transient response analyses. Thus, the computational efficiency of the proposed fast MPC method has been improved a few orders of magnitude than that of the original standard MPC1 and MPC2. At last, a similar control performance is obtained between the proposed fast MPC and the original standard MPC1. But compared with the original standard MPC2, the control performance of the proposed fast MPC has been increased by 5\%-30\% under the same sampling steps and predictive horizons.

The remainder of this paper is organized as follows: in section 2, the standard MPC for large-scale structures with actuator saturation is briefly reviewed and reformulated; in section 3, the fast MPC for large-scale structures with actuator saturation is proposed based on the explicit expression form of the Newmark- $\beta$ method and parametric variational principle; in section 4 , the comparison between the standard MPC and the proposed fast MPC is discussed; in section 5, numerical examples are carried out to verify the validity and high efficiency of the proposed fast MPC; finally, some conclusions are presented in section 6 .

\section{Standard MPC with input saturation}

\subsection{Problem formulation}

The finite element method (FEM) is often used to build the dynamical model of large-scale structures for high-fidelity numerical simulation. In this paper, a linear time-invariant structure that is subjected to an external force and protected by means of a control system with saturation is considered, and the dynamic equation can be written as

$$
\mathbf{M} \ddot{\boldsymbol{q}}(t)+\mathbf{C} \dot{\boldsymbol{q}}(t)+\mathbf{K} \boldsymbol{q}(t)=\mathbf{D}_{1} \mathbf{u}(t)+\mathbf{D}_{2} \mathbf{p}(t)
$$

where $\mathbf{M}, \mathbf{C}$ and $\mathbf{K}$ are the $n \times n$ mass, damping and stiffness matrices, respectively; $\boldsymbol{q}, \dot{\boldsymbol{q}}$ and $\ddot{\boldsymbol{q}}$ are the $n \times 1$ displacement, velocity and acceleration vectors, respectively; $\mathbf{D}_{1}$ and $\mathbf{D}_{2}$ are the location matrix with $n \times m$ dimensions control inputs and the $n \times \bar{m}$ dimensions external force, respectively; $\mathbf{u}$ and $\mathbf{p}$ 
are the $m$-dimensional vector of the control forces and the $\bar{m}$-dimensional vector of the external forces, respectively.

Eq. (1) is obtained by FEM, while for controller design, it is usually transformed into an equation in state space form

$$
\dot{\mathbf{x}}(t)=\mathbf{A x}(t)+\mathbf{B u}(t)+\mathbf{G p}(t)
$$

and

$$
\mathbf{x}=\left\{\begin{array}{l}
\boldsymbol{q} \\
\dot{\boldsymbol{q}}
\end{array}\right\}, \quad \mathbf{A}=\left[\begin{array}{cc}
\mathbf{0} & \mathbf{I}_{1} \\
-\mathbf{M}^{-1} \mathbf{K} & -\mathbf{M}^{-1} \mathbf{C}
\end{array}\right], \mathbf{B}=\left[\begin{array}{c}
\mathbf{0} \\
\mathbf{M}^{-1} \mathbf{D}_{1}
\end{array}\right], \mathbf{G}=\left\{\begin{array}{c}
\mathbf{0} \\
\mathbf{M}^{-1} \mathbf{D}_{2}
\end{array}\right\}
$$

where $\mathbf{I}_{1}$ is an $n \times n$ identity matrix, and the control variable $\mathbf{u}$ is not free, but constrained by the inequality

$$
\boldsymbol{\mu}_{\min } \leq \mathbf{u} \leq \boldsymbol{\mu}_{\max }
$$

where $\boldsymbol{\mu}_{\min } \in R^{m \times 1}$ and $\boldsymbol{\mu}_{\max } \in R^{m \times 1}$ are the lower and upper limits of the control variable $\mathbf{u}$, respectively.

Remark 1: Control actuation devices are subject to saturation. Force, torque, thrust, stroke, voltage, and every conceivable physical input in every conceivable application of control technology are ultimately limited. For instance, a double pusher magneto-rheological fluid damping device can generally provide a maximal damping force of 20 tons.

In real civil engineering, we can only obtain the information of some limited measured points of the structure. Meanwhile, the measured point distributions have much influence on the effect of MPC control. In this paper, we assume that the number and distributions of observation points have been given and the research of observation points and their distributions are out of the subject of the present study. Therefore, the output equation with known observation can be written as

$$
\mathbf{y}=\overline{\mathbf{C}} \mathbf{x}
$$

where $\mathbf{y}$ is the $p \times 1$ output vector, and $\overline{\mathbf{C}}$ is the $p \times 2 n$ observation output coefficient matrix; $p$ is the number of output variables. Finally, substitute the above output equation into the performance index

$$
\mathbf{J}=\frac{1}{2} \int_{t_{0}}^{t_{0}+T}\left(\mathbf{y}^{\mathrm{T}} \overline{\mathbf{Q}} \mathbf{y}+\mathbf{u}^{\mathrm{T}} \overline{\mathbf{R}} \mathbf{u}\right) \mathrm{d} t
$$

where $t_{0}$ is the initial control implementation time for every sampling point; $T$ is the prediction horizon, which should be longer than the sampling period; and $\overline{\mathbf{Q}} \in R^{p \times p}$ and $\overline{\mathbf{R}} \in R^{m \times m}$ are the 
nonnegative and positive definite symmetric weighting matrices, respectively. The performance index $\mathbf{J}$ is widely adopted in vibration control systems [2, 26-27, 32], ensuring that the dynamical responses will be cut down and the energy consumption will be reduced. So far, the standard MPC with input saturation for the structural control problem has been expressed by the above Eqs. (2), (4) and (6).

Remark 2: Because weighting matrices $\overline{\mathbf{Q}}$ and $\overline{\mathbf{R}}$ greatly affect the system performance, some methods have been developed for their determination. Such as trial-and-error method, Bryson's rule and parameter optimization, etc. In this paper, we mainly choose weighting matrices $\overline{\mathbf{Q}}$ and $\overline{\mathbf{R}}$ according to the Bryson's rule, i.e., weighting matrices $\overline{\mathbf{Q}}$ and $\overline{\mathbf{R}}$ are taken as diagonal matrices, and the value of the diagonal elements are determined by the maximum acceptable value of output $\mathbf{y}$ and control $\mathbf{u}$.

\subsection{Quadratic programming method}

Sub-section 2.1 describes the MPC problem based on the continuous dynamic equation. However, MPC is essentially a control algorithm based on a discrete-time system. The continuous time should be divided into a series of time intervals, and then the MPC problem can be transformed into an optimization problem. For standard MPC, the future states can be predicted using the convolution integral $[2,32]$ or the forward Euler differential. In this paper, these two discretization schemes are named the standard MPC1 and the standard MPC2, respectively. Assuming that the number of prediction points is $N$, and the length of the prediction step is $\eta=T / N$. Then, ignoring the external excitation, the solution in discrete form of the state Eq. (2) can be obtained as

$$
\mathbf{x}_{k}=\overline{\mathbf{h}} \mathbf{x}_{k-1}+\overline{\mathbf{w}} \mathbf{u}_{k-1}
$$

Where $\mathbf{u}_{k-1}$ is the control input at discrete-time point $t_{k-1}, \mathbf{x}_{k}$ and $\mathbf{x}_{k-1}$ are the state variables at discrete-time points $t_{k}$ and $t_{k-1}$, respectively. The detailed expressions of matrices $\overline{\mathbf{h}}$ and $\overline{\mathbf{w}}$ are given as follows:

For standard MPC1:

$$
\overline{\mathbf{h}}=\mathrm{e}^{\mathbf{A} \eta}, \overline{\mathbf{w}}=\mathbf{A}^{-1}\left(\mathrm{e}^{\mathbf{A} \eta}-\mathbf{I}_{2}\right) \mathbf{B}
$$

For standard MPC2:

$$
\overline{\mathbf{h}}=\mathbf{I}_{2}+\eta \mathbf{A}, \quad \overline{\mathbf{w}}=\eta \mathbf{B}
$$

Where $\mathbf{I}_{2}$ is a $2 n \times 2 n$ identity matrix.

Remark 3: The discretization scheme Eq. (8) involves much computation of matrix exponentials and 
their integration. Therefore, the computation work will be very time-consuming and memory-consuming or even impossible to be realized, especially for large-scale problems.

Then by performing the recurrence computation of Eq.(7), the future states for all prediction points can be obtained by

$$
\mathbf{x}_{k}=\overline{\mathbf{h}}^{k} \mathbf{x}_{0}+\sum_{j=1}^{k} \overline{\mathbf{h}}^{k-j} \overline{\mathbf{w}} \mathbf{u}_{j-1}, 1 \leq k \leq N
$$

Substituting the above equation into the output Eq.(5), the future outputs for all prediction points can be expressed as

$$
\mathbf{y}_{k}=\overline{\mathbf{C}} \overline{\mathbf{h}}^{k} \mathbf{x}_{0}+\sum_{j=1}^{k} \overline{\mathbf{C}} \overline{\mathbf{h}}^{k-j} \overline{\mathbf{w}} \mathbf{u}_{j-1}, \quad 1 \leq k \leq N
$$

The above Eq. (11) can be rewritten in matrix form as

$$
\begin{aligned}
\mathbf{Y} & =\overline{\mathbf{F}} \mathbf{x}_{0}+\overline{\mathbf{G}} \mathbf{U} \\
& =\left[\begin{array}{c}
\overline{\mathbf{C}} \overline{\mathbf{h}} \\
\overline{\mathbf{C}}^{2} \\
\vdots \\
\overline{\mathbf{C}} \overline{\mathbf{h}}^{N}
\end{array}\right] \mathbf{x}_{0}+\left[\begin{array}{cccc}
\overline{\mathbf{C}} \overline{\mathbf{w}} & \mathbf{0} & \cdots & \mathbf{0} \\
\overline{\mathbf{C}} \overline{\mathbf{h}} \overline{\mathbf{w}} & \overline{\mathbf{C}} \overline{\mathbf{w}} & \ldots & \mathbf{0} \\
\vdots & \vdots & \ddots & \vdots \\
\overline{\mathbf{C}} \overline{\mathbf{h}}^{N-1} \overline{\mathbf{w}} & \overline{\mathbf{C}} \overline{\mathbf{h}}^{N-2} \overline{\mathbf{w}} & \cdots & \overline{\mathbf{C}} \overline{\mathbf{w}}
\end{array}\right]\left\{\begin{array}{c}
\mathbf{u}_{0} \\
\mathbf{u}_{1} \\
\vdots \\
\mathbf{u}_{N-1}
\end{array}\right\}
\end{aligned}
$$

where $\mathbf{Y}=\left[\mathbf{y}_{1}^{\mathrm{T}}, \mathbf{y}_{2}^{\mathrm{T}}, \cdots, \mathbf{y}_{N}^{\mathrm{T}}\right]^{\mathrm{T}}$ and $\mathbf{U}=\left[\mathbf{u}_{0}^{\mathrm{T}}, \mathbf{u}_{1}^{\mathrm{T}}, \cdots, \mathbf{u}_{N-1}^{\mathrm{T}}\right]^{\mathrm{T}}$ are the sequences of the future outputs and optimal control inputs for all prediction points, respectively. The detailed explanation about input horizon can be found in Appendix-A. Then, the performance index expressed in Eq. (6) can be rewritten as follows:

$$
\mathbf{J}=\frac{1}{2} \sum_{i=1}^{N}\left(\mathbf{y}_{i}{ }^{\mathrm{T}} \overline{\mathbf{Q}} \mathbf{y}_{i}+\mathbf{u}_{i}{ }^{\mathrm{T}} \overline{\mathbf{R}} \mathbf{u}_{i}\right)=\frac{1}{2} \mathbf{Y}^{\mathrm{T}} \mathbf{Q Y}+\frac{1}{2} \mathbf{U}^{\mathrm{T}} \mathbf{R} \mathbf{U}
$$

where $\mathbf{Q} \in R^{N p \times N p}$ and $\mathbf{R} \in R^{N m \times N m}$, given as

$$
\mathbf{Q}=\operatorname{diag}(\overline{\mathbf{Q}}, \overline{\mathbf{Q}}, \cdots, \overline{\mathbf{Q}}), \mathbf{R}=\operatorname{diag}(\overline{\mathbf{R}}, \overline{\mathbf{R}}, \cdots, \overline{\mathbf{R}})
$$

Substitute Eq.(12) into the above Eq.(13) and ignore the constant term, and then the performance index can be obtained as follows:

$$
\mathbf{J}\left(\mathbf{U}, \mathbf{x}_{0}\right)=\frac{1}{2} \mathbf{U}^{\mathrm{T}}\left(\overline{\mathbf{G}}^{\mathrm{T}} \mathbf{Q} \overline{\mathbf{G}}+\mathbf{R}\right) \mathbf{U}+\left(\overline{\mathbf{G}}^{\mathrm{T}} \mathbf{Q} \overline{\mathbf{F}} \mathbf{x}_{0}\right)^{\mathrm{T}} \mathbf{U}
$$

Thus, the standard MPC with input saturation problem expressed by Eqs. (2), (4) and (6) can be transformed into a quadratic programming $(\mathrm{QP})$ problem, which can be expressed as 


$$
\left\{\begin{array}{l}
\min _{\mathbf{U}} \frac{1}{2} \mathbf{U}^{\mathrm{T}}\left(\overline{\mathbf{G}}^{\mathrm{T}} \mathbf{Q} \overline{\mathbf{G}}+\mathbf{R}\right) \mathbf{U}+\left(\overline{\mathbf{G}}^{\mathrm{T}} \mathbf{Q} \overline{\mathbf{F}} \mathbf{x}_{0}\right)^{\mathrm{T}} \mathbf{U} \\
\text { s.t. } \overline{\mathbf{E}} \boldsymbol{\mu}_{\min } \leq \mathbf{U} \leq \overline{\mathbf{E}} \boldsymbol{\mu}_{\max }
\end{array}\right.
$$

where $\overline{\mathbf{E}}$ is an $m N \times m$ matrix for enlarged dimensions, given as

$$
\overline{\mathbf{E}}=\left[\mathbf{I}_{m}^{\mathrm{T}}, \mathbf{I}_{m}^{\mathrm{T}}, \cdots, \mathbf{I}_{m}^{\mathrm{T}}\right]^{\mathrm{T}}
$$

where $\mathbf{I}_{m}$ is an $m \times m$ identity matrix.

Remark 4: Eq. (16) is a standard quadratic programming problem and can be solved by many optimization solvers. Quadprog is a script function that can be called directly in MATLAB software. There are three different algorithms for its optimization, that is, the interior-point-convex quadprog algorithm [40], trust-region-reflective quadprog algorithm [41], and active-set quadprog algorithm [42], and the first one is specified in this paper. QPC is the abbreviation "quadratic programming in C" [43], and it was coded by Brett Ninness and Adrian Wills from the University of Newcastle. The efficiency of QPC is much faster than the standard solvers including Quadprog. In this paper, both QPC and Quadprog are used to solve the quadratic programming problem for comparison.

\section{Proposed fast MPC with input saturation}

In this section, based on the second-order continuous dynamic equation, a new explicit MPC structure with actuator saturation is proposed from a structural dynamics perspective. Then, according to the proposed explicit MPC structure, a valid and high-efficiency algorithm is also developed for the practical computation of large-scale structures. The detailed depiction and formula derivation can be found in the following sub-sections.

\subsection{Explicit expression form for the Newmark- $\beta$ method}

In the previous section, the dynamic Eq. (1) without external force can be rewritten as

$$
\mathbf{M} \ddot{\boldsymbol{q}}(t)+\mathbf{C} \dot{\boldsymbol{q}}(t)+\mathbf{K} \boldsymbol{q}(t)=\mathbf{D}_{1} \mathbf{u}(t)
$$

In the Newmark- $\beta$ method, the basic assumptions for the velocity and displacement [44] are

$$
\begin{gathered}
\dot{\boldsymbol{q}}_{k+1}=\dot{\boldsymbol{q}}_{k}+\left[(1-\delta) \ddot{\boldsymbol{q}}_{k}+\delta \ddot{\boldsymbol{q}}_{k+1}\right] \Delta t \\
\boldsymbol{q}_{k+1}=\boldsymbol{q}_{k}+\dot{\boldsymbol{q}}_{k} \Delta t+\left[\left(\frac{1}{2}-\alpha\right) \ddot{\boldsymbol{q}}_{k}+\alpha \ddot{\boldsymbol{q}}_{k+1}\right](\Delta t)^{2}
\end{gathered}
$$

where $\Delta t$ is the step length and $\delta$ and $\alpha\left(\delta \geq 0.5, \alpha \geq 0.25(0.5+\delta)^{2}\right)$ are important parameters that determine the stability and accuracy of the Newmark- $\beta$ method. At time $t_{k+1}=t_{k}+\Delta t$, the 
dynamic responses (displacement, velocity and acceleration) must satisfy the dynamic balance equation, given by

$$
\mathbf{M} \ddot{\boldsymbol{q}}_{k+1}+\mathbf{C} \dot{\boldsymbol{q}}_{k+1}+\mathbf{K} \boldsymbol{q}_{k+1}=\mathbf{D}_{1} \mathbf{u}_{k+1}
$$

Then, combining Eqs. (19), (20) and (21), the dynamic responses at time $t_{k+1}$ can be obtained from the dynamic responses at time $t_{k}$, given as

$$
\begin{gathered}
\boldsymbol{q}_{\mathrm{k}+1}=\hat{\mathbf{K}}^{-1} \mathbf{D}_{1} \mathbf{u}_{\mathrm{k}+1}+\Psi_{11} \boldsymbol{q}_{\mathrm{k}}+\Psi_{12} \dot{\boldsymbol{q}}_{\mathrm{k}}+\Psi_{13} \ddot{\boldsymbol{q}}_{\mathrm{k}} \\
\dot{\boldsymbol{q}}_{\mathrm{k}+1}=\mathrm{b}_{1} \hat{\mathbf{K}}^{-1} \mathbf{D}_{1} \mathbf{u}_{\mathrm{k}+1}+\Psi_{21} \boldsymbol{q}_{\mathrm{k}}+\Psi_{22} \dot{\boldsymbol{q}}_{\mathrm{k}}+\Psi_{23} \ddot{\boldsymbol{q}}_{\mathrm{k}} \\
\ddot{\boldsymbol{q}}_{\mathrm{k}+1}=\mathrm{b}_{4} \hat{\mathbf{K}}^{-1} \mathbf{D}_{1} \mathbf{u}_{\mathrm{k}+1}+\Psi_{31} \boldsymbol{q}_{\mathrm{k}}+\Psi_{32} \dot{\boldsymbol{q}}_{\mathrm{k}}+\Psi_{33} \ddot{\boldsymbol{q}}_{\mathrm{k}}
\end{gathered}
$$

Where $\hat{\mathbf{K}}$ is called the effective stiffness matrix

$$
\hat{\mathbf{K}}=\mathrm{b}_{4} \mathbf{M}+\mathrm{b}_{1} \mathbf{C}+\mathbf{K}
$$

The detailed expressions for coefficient $\Psi_{\mu v}(\mu, v=1,2,3)$ and $\mathrm{b}_{\kappa}(\kappa=1,2,3,4,5,6)$ in Eq.(22)-(24) can be found in Appendix-B. Let the dynamic response $\mathbf{v}=\left[\boldsymbol{q}^{\mathrm{T}}, \dot{\boldsymbol{q}}^{\mathrm{T}}, \ddot{\boldsymbol{q}}^{\mathrm{T}}\right]^{\mathrm{T}}$, and those at times $t_{k}$ and $t_{k+1}$ can be expressed as

$$
\mathbf{v}_{k}=\left\{\begin{array}{l}
\boldsymbol{q}_{k} \\
\dot{\boldsymbol{q}}_{k} \\
\ddot{\boldsymbol{q}}_{k}
\end{array}\right\}, \quad \mathbf{v}_{k+1}=\left\{\begin{array}{l}
\boldsymbol{q}_{k+1} \\
\dot{\boldsymbol{q}}_{k+1} \\
\ddot{\boldsymbol{q}}_{k+1}
\end{array}\right\}
$$

Then, substituting Eqs. (22), (23) and (24) into the above Eq. (26), the recurrence formula for the Newmark- $\beta$ method can be obtained as

$$
\mathbf{v}_{k+1}=\mathbf{h} \mathbf{v}_{k}+\mathbf{w} \mathbf{u}_{k+1}
$$

where

$$
\mathbf{h}=\left[\begin{array}{lll}
\Psi_{11} & \Psi_{12} & \Psi_{13} \\
\Psi_{21} & \Psi_{22} & \Psi_{23} \\
\Psi_{31} & \Psi_{32} & \Psi_{33}
\end{array}\right], \quad \mathbf{W}=\left[\begin{array}{c}
\hat{\mathbf{K}}^{-1} \mathbf{D}_{1} \\
\mathrm{~b}_{1} \hat{\mathbf{K}}^{-1} \mathbf{D}_{1} \\
\mathrm{~b}_{4} \hat{\mathbf{K}}^{-1} \mathbf{D}_{1}
\end{array}\right]
$$

Then, substituting the initial state $\mathbf{x}_{0}=\left[\boldsymbol{q}_{0}^{\mathrm{T}}, \dot{\boldsymbol{q}}_{0}^{\mathrm{T}}\right]^{\mathrm{T}}$ into Eq. (21) at time $t_{0}$, the initial acceleration $\ddot{\boldsymbol{q}}_{0}$ can be computed. Thus, the initial dynamic response $\mathbf{v}_{0}=\left[\boldsymbol{q}_{0}^{\mathrm{T}}, \dot{\boldsymbol{q}}_{0}^{\mathrm{T}}, \ddot{\boldsymbol{q}}_{0}^{\mathrm{T}}\right]^{\mathrm{T}}$ can be obtained as

$$
\mathbf{v}_{0}=g_{1} \mathbf{x}_{0}+g_{2} \mathbf{u}_{0}
$$

where 


$$
\boldsymbol{g}_{1}=\left[\begin{array}{cc}
\mathbf{I}_{1} & \mathbf{0} \\
\mathbf{0} & \mathbf{I}_{1} \\
-\mathbf{M}^{-1} \mathbf{K} & -\mathbf{M}^{-1} \mathbf{C}
\end{array}\right], \quad \boldsymbol{g}_{2}=\left[\begin{array}{c}
\mathbf{0} \\
\mathbf{0} \\
\mathbf{M}^{-1} \mathbf{D}_{1}
\end{array}\right]
$$

By performing an iterative computation of Eq. (27), the future dynamic responses for all time steps can be obtained as

$$
\mathbf{v}_{k}=\hat{\mathbf{H}}_{k} \mathbf{x}_{0}+\sum_{j=0}^{k} \hat{\mathbf{W}}_{k, j} \mathbf{u}_{j}, \quad 1 \leq k \leq N
$$

where

$$
\hat{\mathbf{H}}_{k}=\mathbf{h}^{k} \boldsymbol{g}_{1}, \hat{\mathbf{W}}_{k, j}=\left\{\begin{array}{lr}
\mathbf{h}^{k} \boldsymbol{g}_{2}, & j=0 \\
\mathbf{h}^{k-j} \mathbf{w}, & 1 \leq j \leq k
\end{array}\right.
$$

Eq. (30) can be rewritten in matrix form,

$$
\begin{aligned}
\mathbf{V} & =\hat{\mathbf{H}} \mathbf{x}_{0}+\hat{\mathbf{W}} \mathbf{u} \\
& =\left[\begin{array}{c}
\mathbf{h} \boldsymbol{g}_{1} \\
\mathbf{h}^{2} \boldsymbol{g}_{1} \\
\mathbf{h}^{3} \boldsymbol{g}_{1} \\
\vdots \\
\mathbf{h}^{N} \boldsymbol{g}_{1}
\end{array}\right] \mathbf{x}_{0}+\left[\begin{array}{ccccc}
\mathbf{h} \boldsymbol{g}_{2} & \mathbf{w} & \mathbf{0} & \cdots & \mathbf{0} \\
\mathbf{h}^{2} \boldsymbol{g}_{2} & \mathbf{h w} & \mathbf{w} & \cdots & \mathbf{0} \\
\mathbf{h}^{3} \boldsymbol{g}_{2} & \mathbf{h}^{2} \mathbf{w} & \mathbf{h w} & \cdots & \mathbf{0} \\
\vdots & \vdots & \vdots & \ddots & \vdots \\
\mathbf{h}^{N} \boldsymbol{g}_{2} & \mathbf{h}^{N-1} \mathbf{w} & \mathbf{h}^{N-2} \mathbf{w} & \cdots & \mathbf{w}
\end{array}\right]\left\{\begin{array}{c}
\mathbf{u}_{0} \\
\mathbf{u}_{1} \\
\mathbf{u}_{2} \\
\vdots \\
\mathbf{u}_{N}
\end{array}\right\}
\end{aligned}
$$

where $\mathbf{V}$ is the set of all the future dynamic responses.

Remark 5: The future system output $\mathbf{V}$ is explicitly represented as a function of the initial state and the control forces. Equation (32) is regarded as the explicit expression form of the Newmark- $\beta$ method in this paper. The difference between the traditional Newmark- $\beta$ method and the present explicit expression form is that the former is expressed by the second-order structural dynamics equation and the latter is described by the first-order state space equation.

\subsection{Physical meaning and computation of matrices $\hat{\mathbf{W}}$ and $\hat{\mathbf{H}} \mathbf{x}_{0}$}

For the explicit expression form of the Newmark- $\beta$ method, as shown in Eq. (32), the future dynamic responses $\mathbf{V}$ consist of two parts. On the one hand, $\mathbf{V}$ is derived from the initial state of the system, and on the other hand, it is derived from the control forces. For further study and for exact and convenient expression, we can call matrix $\mathbf{h}$ in Eq. (31) the state transition matrix, and then the main physical meaning of the explicit expression form of the Newmark- $\beta$ method can be expressed as two points.

First, the physical meaning of matrix $\hat{\mathbf{W}}_{k, j}$ is the dynamic responses at time $t_{k}$ 
$(k=1,2,3, \ldots, N$ and $k \geq j)$, which is caused by the unit control input at time $t_{j}(j=0,1,2, \ldots, N)$. The responses of the adjacent moment can be easily deduced through the state transition matrix $\mathbf{h}$, given by $\hat{\mathbf{W}}_{k+1, j}=\mathbf{h} \hat{\mathbf{W}}_{k, j}$. Second, the physical meaning of matrix $\hat{\mathbf{H}}_{k}$ is the dynamic responses derived from the unit initial state, and the responses of the adjacent moment can also be deduced analogously, given as $\hat{\mathbf{H}}_{k+1}=\mathbf{h} \hat{\mathbf{H}}_{k}$. Because the structure is a linear system, then based on the superposition principle, the dynamic responses caused by the initial state $\mathbf{x}_{0}$ is $\hat{\mathbf{H}}_{k} \mathbf{x}_{0}$. Likewise, the dynamic responses caused by the control input $\mathbf{u}_{j}$ is $\sum_{j=0}^{k} \hat{\mathbf{W}}_{k, j} \mathbf{u}_{j}$.

Then, according to the above analysis of the explicit expression form of the Newmark- $\beta$ method, the matrixes $\hat{\mathbf{W}}$ and $\hat{\mathbf{H}} \mathbf{x}_{0}$ can be computed as follows:

\section{(1) Computation for $\hat{\mathbf{W}}$}

As seen from Eq. (32), only the first two block-columns ( $\hat{\mathbf{W}}_{k, 1}$ and $\hat{\mathbf{W}}_{k, 2}$ ) of $\hat{\mathbf{W}}$ should be computed, while the other block-columns can be easily obtained from $\hat{\mathbf{W}}_{k, 2}$. Moreover, according to the physical meaning of matrix $\hat{\mathbf{W}}_{k, j}$, let the initial state $\mathbf{x}_{0}=\mathbf{0}$ and apply unit control input $\mathbf{u}_{0}=\mathbf{I}_{\mathrm{m}}$ to the dynamic system at time $t_{0}$. Then, the dynamic responses for all time steps can be obtained as follows:

$$
\mathbf{V}=\left[\begin{array}{ccccc}
\mathbf{h} \boldsymbol{g}_{2} & \mathbf{w} & \mathbf{0} & \cdots & \mathbf{0} \\
\mathbf{h}^{2} \boldsymbol{g}_{2} & \mathbf{h w} & \mathbf{w} & \cdots & \mathbf{0} \\
\mathbf{h}^{3} \boldsymbol{g}_{2} & \mathbf{h}^{2} \mathbf{w} & \mathbf{h w} & \cdots & \mathbf{0} \\
\vdots & \vdots & \vdots & \ddots & \vdots \\
\mathbf{h}^{N} \boldsymbol{g}_{2} & \mathbf{h}^{N-1} \mathbf{w} & \mathbf{h}^{N-2} \mathbf{w} & \cdots & \mathbf{w}
\end{array}\right]\left\{\begin{array}{l}
\mathbf{I}_{\mathrm{m}} \\
\mathbf{0} \\
\mathbf{0} \\
\vdots \\
\mathbf{0}
\end{array}\right\}=\left[\begin{array}{c}
\mathbf{h} \boldsymbol{g}_{2} \\
\mathbf{h}^{2} \boldsymbol{g}_{2} \\
\mathbf{h}^{3} \boldsymbol{g}_{2} \\
\vdots \\
\mathbf{h}^{N} \boldsymbol{g}_{2}
\end{array}\right]=\hat{\mathbf{W}}_{k, 1}
$$

In a similar way, if we let the initial state $\mathbf{x}_{0}=\mathbf{0}$, and apply unit control input $\mathbf{u}_{1}=\mathbf{I}_{\mathrm{m}}$ to the dynamic system at time $t_{1}$, then the dynamic responses for all time steps can be obtained as follows:

$$
\mathbf{V}=\left[\begin{array}{ccccc}
\mathbf{h} \boldsymbol{g}_{2} & \mathbf{w} & \mathbf{0} & \ldots & \mathbf{0} \\
\mathbf{h}^{2} \boldsymbol{g}_{2} & \mathbf{h w} & \mathbf{w} & \ldots & \mathbf{0} \\
\mathbf{h}^{3} \boldsymbol{g}_{2} & \mathbf{h}^{2} \mathbf{w} & \mathbf{h w} & \ldots & \mathbf{0} \\
\vdots & \vdots & \vdots & \ddots & \vdots \\
\mathbf{h}^{N} \boldsymbol{g}_{2} & \mathbf{h}^{N-1} \mathbf{w} & \mathbf{h}^{N-2} \mathbf{w} & \cdots & \mathbf{w}
\end{array}\right]\left\{\begin{array}{c}
\mathbf{0} \\
\mathbf{I}_{\mathrm{m}} \\
\mathbf{0} \\
\vdots \\
\mathbf{0}
\end{array}\right\}=\left[\begin{array}{c}
\mathbf{w} \\
\mathbf{h w} \\
\mathbf{h}^{2} \mathbf{w} \\
\vdots \\
\mathbf{h}^{N-1} \mathbf{w}
\end{array}\right]=\hat{\mathbf{W}}_{k, 2}
$$


Eqs. (33) and (34) mean that $\hat{\mathbf{W}}_{k, 1}$ and $\hat{\mathbf{W}}_{k, 2}$ can both be obtained by carrying out one transient analysis on the dynamic system using the Newmark- $\beta$ method with a zero initial state and unit control input at times $t_{0}$ and $t_{1}$.

(2) Computation for $\hat{\mathbf{H}} \mathbf{x}_{0}$

According to the physical meaning of matrix $\hat{\mathbf{H}}_{k}$, when only an initial state $\mathbf{x}_{0}$ is applied to the system and with no input, the dynamic responses for all time steps can be obtained as follows:

$$
\mathbf{V}=\hat{\mathbf{H}} \mathbf{x}_{0}
$$

This means that $\hat{\mathbf{H}} \mathbf{x}_{0}$ can also be computed by carrying out one transient analysis on the dynamic system using the Newmark- $\beta$ method with the initial state $\mathbf{x}_{0}$ and no input.

\subsection{Parametric variational principle for MPC with input saturation}

The above two sub-sections give the fast MPC without input saturation. In this sub-section, based on the parametric variational principle [45], the fast MPC with input saturation will be proposed, and then a linear complementary problem will be developed to replace the quadratic programming problem.

First, substituting the above Eq. (30) into Eq. (5), the future outputs for all prediction points can be obtained as

$$
\mathbf{y}_{k}=\hat{\mathbf{C}} \hat{\mathbf{H}}_{k} \mathbf{x}_{0}+\sum_{j=0}^{k} \hat{\mathbf{C}} \hat{\mathbf{W}}_{k, j} \mathbf{u}_{j}, \quad 1 \leq k \leq N
$$

where $\hat{\mathbf{C}}=\left[\begin{array}{ll}\overline{\mathbf{C}} & \mathbf{0}\end{array}\right]$ is a $p \times 3 n$ matrix, which can be rewritten as follows:

$$
\begin{aligned}
\mathbf{Y} & =\mathbf{H} \mathbf{x}_{0}+\mathbf{W U} \\
& =\left[\begin{array}{c}
\hat{\mathbf{C}} \mathbf{h} \boldsymbol{g}_{1} \\
\hat{\mathbf{C}} \mathbf{h}^{2} \boldsymbol{g}_{1} \\
\hat{\mathbf{C}} \mathbf{h}^{3} \boldsymbol{g}_{1} \\
\vdots \\
\hat{\mathbf{C}} \mathbf{h}^{N} \boldsymbol{g}_{1}
\end{array}\right] \mathbf{x}_{0}+\left[\begin{array}{ccccc}
\hat{\mathbf{C}} \boldsymbol{g}_{2} & \hat{\mathbf{C}} \mathbf{w} & \mathbf{0} & \cdots & \mathbf{0} \\
\hat{\mathbf{C}} \mathbf{h}^{2} \boldsymbol{g}_{2} & \hat{\mathbf{C}} \mathbf{h w} & \hat{\mathbf{C}} \mathbf{w} & \cdots & \mathbf{0} \\
\hat{\mathbf{C}} \mathbf{h}^{3} \boldsymbol{g}_{2} & \hat{\mathbf{C}} \mathbf{h}^{2} \mathbf{w} & \hat{\mathbf{C}} \mathbf{h w} & \cdots & \mathbf{0} \\
\vdots & \vdots & \vdots & \ddots & \vdots \\
\hat{\mathbf{C}} \mathbf{h}^{N} \boldsymbol{g}_{2} & \hat{\mathbf{C}} \mathbf{h}^{N-1} \mathbf{w} & \hat{\mathbf{C}} \mathbf{h}^{N-2} \mathbf{w} & \cdots & \hat{\mathbf{C}} \mathbf{w}
\end{array}\right]\left\{\begin{array}{c}
\mathbf{u}_{0} \\
\mathbf{u}_{1} \\
\mathbf{u}_{2} \\
\vdots \\
\mathbf{u}_{N}
\end{array}\right\}
\end{aligned}
$$

Second, introducing variables $\bar{\alpha}$ and $\underline{\alpha}$, the inequality constraints in Eq. (16) can be transformed into equality constraints as follows:

$$
\left\{\begin{array}{l}
\mathbf{U}-\overline{\mathbf{E}} \mu_{\max }+\bar{\alpha}=\mathbf{0} \\
\overline{\mathbf{E}} \boldsymbol{\mu}_{\min }-\mathbf{U}+\underline{\alpha}=\mathbf{0}
\end{array}\right.
$$


According to the parametric variational principle [45], an expanded performance index $\mathbf{J}_{\mathrm{e}}$ can be given as

$$
\mathbf{J}_{\mathrm{e}}=\frac{1}{2} \mathbf{Y}^{\mathrm{T}} \mathbf{Q Y}+\frac{1}{2} \mathbf{U}^{\mathrm{T}} \mathbf{R} \mathbf{U}+\overline{\boldsymbol{\lambda}}^{\mathrm{T}}\left(\mathbf{U}-\overline{\mathbf{E}} \boldsymbol{\mu}_{\max }+\overline{\boldsymbol{\alpha}}\right)+\underline{\boldsymbol{\lambda}}^{\mathrm{T}}\left(\overline{\mathbf{E}} \boldsymbol{\mu}_{\min }-\mathbf{U}+\underline{\boldsymbol{\alpha}}\right)
$$

where $\bar{\lambda}$ and $\underline{\lambda}$ are the Lagrange multipliers. Substituting the discrete dynamic Eq. (37) into the above equation, the problem is changed into an unconstrained optimization problem. Performing the calculus of variations with respect to the control variables $\mathbf{U}$ gives

$$
\frac{\partial \mathbf{J}_{\mathrm{e}}}{\partial \mathbf{U}}=0
$$

The control variables $\mathbf{U}$ can be explicitly expressed by the above equation, given as

$$
\mathbf{U}=-\left(\mathbf{W}^{\mathrm{T}} \mathbf{Q W}+\mathbf{R}\right)^{-1}\left(\mathbf{W}^{\mathrm{T}} \mathbf{Q} \mathbf{H} \mathbf{x}_{0}+\bar{\lambda}-\underline{\lambda}\right)
$$

To avoid the reduplicative computation of matrixes, let

$$
\left\{\begin{array}{l}
\overline{\mathbf{K}}_{1}=\left(\mathbf{W}^{\mathrm{T}} \mathbf{Q W}+\mathbf{R}\right)^{-1} \\
\overline{\mathbf{K}}_{2}=\overline{\mathbf{K}}_{1} \mathbf{W}^{\mathrm{T}} \mathbf{Q}
\end{array}\right.
$$

Then, Eq. (41) can be rewritten as follows:

$$
\mathbf{U}=-\overline{\mathbf{K}}_{1}(\bar{\lambda}-\underline{\lambda})-\overline{\mathbf{K}}_{2} \mathbf{H} \mathbf{x}_{0}
$$

Substituting the above equation into the constraint equation (38), the constraint condition can be transformed as follows:

$$
\left\{\begin{array}{l}
-\overline{\mathbf{K}}_{1}(\bar{\lambda}-\underline{\lambda})-\overline{\mathbf{K}}_{2} \mathbf{H} \mathbf{x}_{0}-\overline{\mathbf{E}} \boldsymbol{\mu}_{\max }+\bar{\alpha}=0 \\
\overline{\mathbf{E}}_{\boldsymbol{\mu}_{\min }}+\overline{\mathbf{K}}_{1}(\bar{\lambda}-\underline{\lambda})+\overline{\mathbf{K}}_{2} \mathbf{H} \mathbf{x}_{0}+\underline{\alpha}=\mathbf{0}
\end{array}\right.
$$

Rewrite the above formula in matrix form, given as

$$
\left\{\begin{array}{c}
-\overline{\mathbf{E}} \boldsymbol{\mu}_{\max }-\overline{\mathbf{K}}_{2} \mathbf{H} \mathbf{x}_{0} \\
\overline{\mathbf{E}} \boldsymbol{\mu}_{\min }+\overline{\mathbf{K}}_{2} \mathbf{H} \mathbf{x}_{0}
\end{array}\right\}+\left[\begin{array}{cc}
-\overline{\mathbf{K}}_{1} & \overline{\mathbf{K}}_{1} \\
\overline{\mathbf{K}}_{1} & -\overline{\mathbf{K}}_{1}
\end{array}\right]\left\{\begin{array}{l}
\bar{\lambda} \\
\underline{\lambda}
\end{array}\right\}+\left\{\begin{array}{l}
\bar{\alpha} \\
\underline{\alpha}
\end{array}\right\}=\mathbf{0}
$$

Finally, the MPC problem with input saturation is transformed into a linear complementary problem, given as

$$
\left\{\begin{array}{l}
\hat{\mathbf{q}}+\Gamma \hat{\mathbf{z}}+\hat{\mathbf{v}}=\mathbf{0} \\
\hat{\mathbf{v}} \geq \mathbf{0}, \hat{\mathbf{z}} \geq \mathbf{0}, \hat{\mathbf{z}}^{\mathrm{T}} \hat{\mathbf{v}}=\mathbf{0}
\end{array}\right.
$$

where 


$$
\hat{\mathbf{q}}=\left\{\begin{array}{l}
-\overline{\mathbf{E}} \boldsymbol{\mu}_{\max }-\overline{\mathbf{K}}_{2} \mathbf{H} \mathbf{x}_{0} \\
\overline{\mathbf{E}} \boldsymbol{\mu}_{\min }+\overline{\mathbf{K}}_{2} \mathbf{H} \mathbf{x}_{0}
\end{array}\right\}, \Gamma=\left[\begin{array}{cc}
-\overline{\mathbf{K}}_{1} & \overline{\mathbf{K}}_{1} \\
\overline{\mathbf{K}}_{1} & -\overline{\mathbf{K}}_{1}
\end{array}\right], \hat{\mathbf{z}}=\left\{\begin{array}{l}
\bar{\lambda} \\
\underline{\lambda}
\end{array}\right\}, \hat{\mathbf{v}}=\left\{\begin{array}{l}
\bar{\alpha} \\
\underline{\alpha}
\end{array}\right\}
$$

The linear complementarity problem can be solved by the interior point method [46], the pivotal method [47], or another method. In this paper, the Lemke algorithm, which belongs to the pivotal method category, is adopted to solve the linear complementarity problem.

Remark 6: Compared with the standard MPC method, the proposed fast MPC method has three different points. First of all, the proposed method is derived directly on the basis of the second-order dynamic equation by using the Newmark- $\beta$ algorithm, rather than the first-order state equation. Second, the discrete dynamic Eq. (37) of the proposed fast MPC method contains the large-scale matrices $\mathbf{H}$ and $\mathbf{W}$, whose formats are similar to those of $\overline{\mathbf{F}}$ and $\overline{\mathbf{G}}$ of Eq. (12), and they are obtained by applying the physical meaning expressed in section 3.2, but not assembled directly as the standard MPC method. Third, the explicit structure of the MPC is obtained by the proposed method. The standard MPC method with input saturation is transferred into a quadratic programming problem and has no obvious structure for a large-scale structural problem. However, the present explicit structure of the MPC can be obtained by solving a linear complementary problem based on the parametric variational principle.

Based on the preceding analysis, the implementation for large-scale structures is summarized as Table 1, and the flow chart of the proposed method is shown in Fig. 1.

Table 1 The implementation for large-scale structures by the proposed fast MPC.

Algorithm: the proposed fast MPC with actuator saturation for large-scale structures

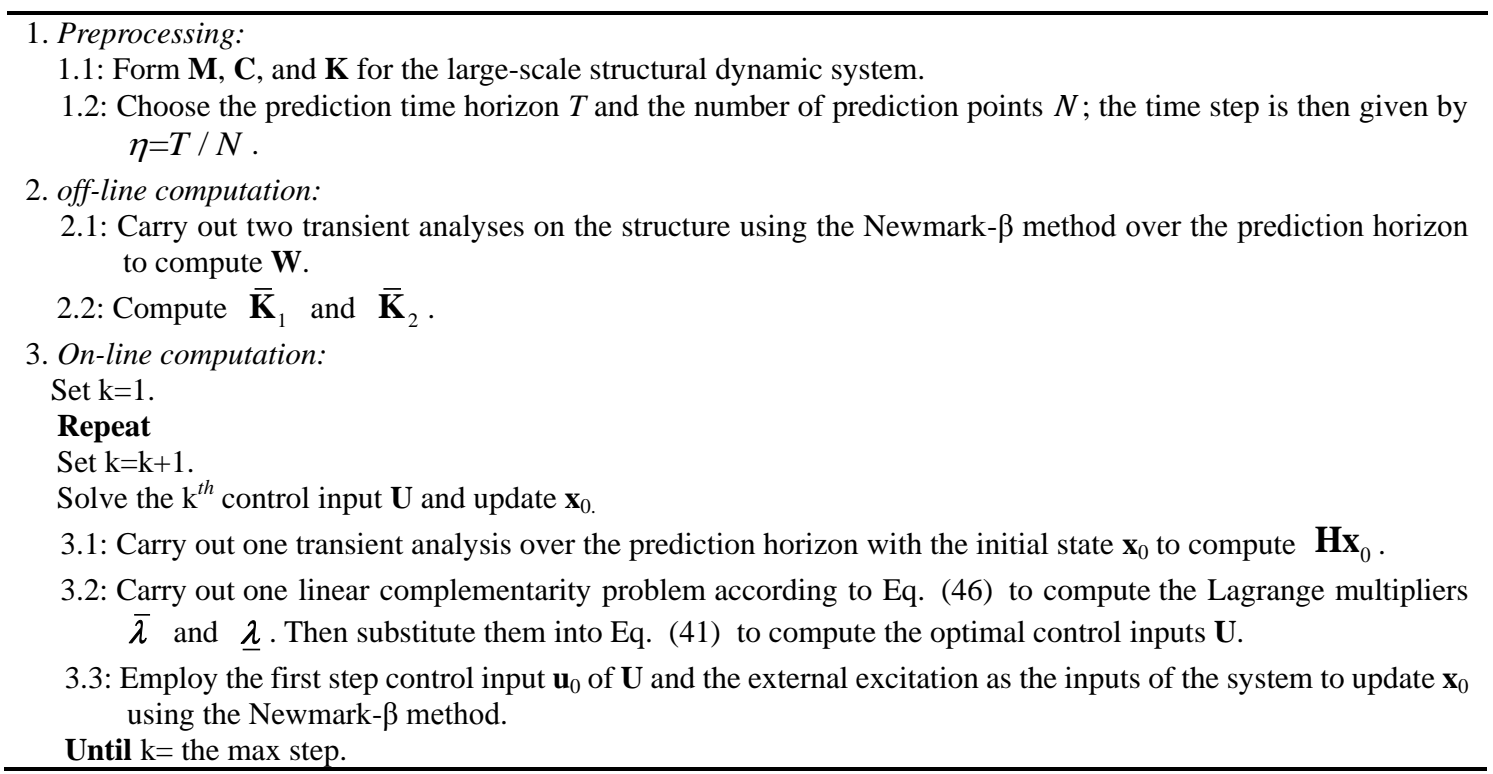




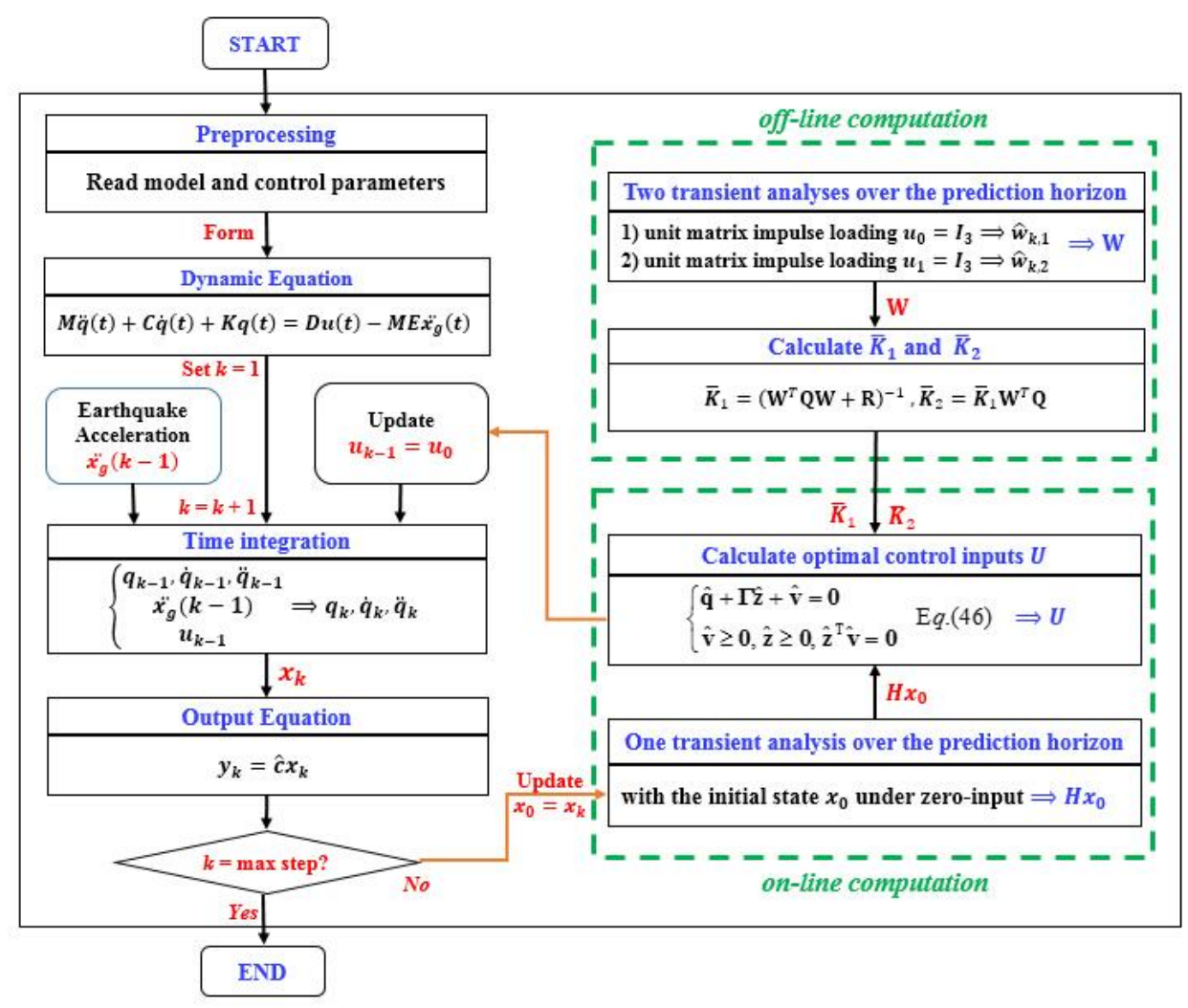

Fig. 1. Flow chart of the proposed fast MPC with input saturation for large-scale dynamic systems.

\section{Comparison between the standard MPC and the proposed fast MPC}

To clearly explain the difference between the standard MPC and the proposed fast MPC, the computational work, memory and efficiency should be illustrated from theory before the numerical simulations.

In general, the standard MPC method is transformed into a quadratic programming problem, and both the QPC and Quadprog are optimization solvers for quadratic programming problems. While the proposed fast MPC method transforms the original problem into a linear complementarity problem to be solved by the Lemke algorithm. For easy comparison, the main computation work for the standard and proposed MPC methods are listed in Table 2.

Table 2 The main computation work of the standard MPC method and the proposed methods.

\begin{tabular}{ll}
\hline \multicolumn{1}{c}{ Standard MPC method } & \multicolumn{1}{c}{ Proposed MPC method } \\
\hline off-line computation & off-line computation \\
(a1) the computations of matrices $\overline{\mathbf{A}}, \overline{\mathbf{B}}$ in (3) & (a2) two transient analyses to compute $\mathbf{W}$ \\
(b1) the computations of matrices $\overline{\mathbf{h}}, \overline{\mathbf{w}}$ in (8) & (b2) the computations of matrices $\overline{\mathbf{k}}_{1}, \overline{\mathbf{k}}_{2}$ in (42) \\
for MPC1 and in (9) for MPC2 & $\begin{array}{l}\text { On-line computation } \\
\text { (c2) one transient analysis to compute } \mathbf{H} \mathbf{x}_{0}\end{array}$ \\
(c1) the assemblies of matrices $\overline{\mathbf{F}}, \overline{\mathbf{G}}$ in (12) & (d2) one linear complementarity problem analysis to \\
On-line computation &
\end{tabular}


\begin{tabular}{ll}
\hline (d1) one quadratic programming problem analysis & $\begin{array}{l}\text { compute Lagrange multipliers and substitute them } \\
\text { into Eq.(41) to compute } \mathbf{U}\end{array}$ \\
\hline
\end{tabular} Compared with the standard MPC method, the advantages of the proposed fast MPC method can be illustrated as follows:

(1) For the length of sampling step:

With the increasing scale of the structural system, the proposed fast MPC method and the standard MPC1 are always suited and valid despite the large length of the sampling step. However, the standard MPC2 can be applied only for small-length sampling steps. Furthermore, the larger the scale of the structural system, the smaller the length of sampling step for the standard MPC2 must be employed, or otherwise the numerical simulation will lose its controlling effect and may even be divergent. For example, in the numerical simulations in the next section, when the structural degrees of freedom is 1890 , to make sure that the numerical solution is not divergent and that the structure system can be controlled efficiently, the sampling period for the standard MPC2 should be 100 times smaller and the predicted horizon should be 500 times smaller than those of the proposed fast MPC method.

(2) For the computation efficiency:

For standard MPC1, as can be seen from Table 2, the major computations for the standard MPC1 are the off-line computation. Moreover, the main implementation of MPC1 concentrates on processes (b1) and (c1). In (b1) of MPC1, the matrix exponential should be computed. Based on the study of Moler and Van Loan (1978), the exponential of a matrix can be computed in many ways, involving approximation theory, differential equations, the matrix eigenvalues, and the matrix characteristic polynomial, but none of them are completely satisfactory. Especially for large-scale problems, the computation work will be very time-consuming and memory-consuming, or even impossible to be realized. For example from the next section, when the number of degrees of freedom $n=3930$, the maximum memory requirement is about $5.13 \mathrm{G}$ and the off-line CPU time is about 2929.94s. In addition, if the input horizon $S$ is defined shorter than the prediction horizon $N$, the off-line CPU time and the maximum memory requirement can still not be reduced effectively. Because even the input horizon has been reduced as short as 1 , it just reduces the scale of some sparse matrix multiplication calculation and their integration. However, the much computation of matrix exponentials have not been avoided, which is very time-consuming and memory-consuming, especially for large-scale problems.

For standard MPC2, on the one hand, to obtain valid control results, the predicted horizon length $\eta$ 
of the standard MPC2 should be set to a very small value, especially for a large-scale structure system. On the other hand, to ensure the feasibility of the general MPC method, the prediction horizon $T$ should not be smaller than the sampling period. Then, the number of prediction points $N$ must increase correspondingly. Though the discrete state based on the forward Euler differencing can avoid large numbers of matrix exponentials, the large number of prediction points will cause the scale of matrixes $\overline{\mathbf{F}}$ and $\overline{\mathbf{G}}$ to increase sharply in process (c1) of Eq. (12). That is to say, a series of matrix-matrix multiplications should be computed, such as $\overline{\mathbf{C}} \overline{\mathbf{h}}, \overline{\mathbf{C}} \overline{\mathbf{h}}^{2}, \ldots, \overline{\mathbf{C}} \overline{\mathbf{h}}^{N}$, and $\overline{\mathbf{C}} \overline{\mathbf{w}}, \overline{\mathbf{C}} \overline{\mathbf{h}} \overline{\mathbf{w}}, \ldots, \overline{\mathbf{C}} \overline{\mathbf{h}}^{N-1} \overline{\mathbf{w}}$. For the above reasons, the computation scale of the quadratic programming problem in process (d1) is also quickly enlarged, especially for large-scale structures. In addition, because the sampling periods of the standard MPC2 are small, a large number of sampling points should be used to complete the whole simulation process. As is shown in the next section, when the sampling period is reduced to $1 / 100$, the number of sampling points will correspondingly increase 100-fold. In a word, the traditional manner for transforming the standard MPC2 problem into a quadratic programming problem and solving it by QPC or Quadprog is very time-consuming and memory-consuming.

For the proposed fast MPC method, it can be seen from the Table 2 that matrices $\mathbf{W}$ and $\mathbf{H} \mathbf{x}_{0}$ can be obtained by two off-line transient analyses and one on-line transient analysis at each sampling instant in processes (a2) and (c2), respectively. Moreover, the proposed fast MPC method can be used with a large sampling step. Therefore, it is very simple and time-saving to build the linear complementary Eq. (46) through assignments and assembling. Obtaining the control inputs by using the Lemke algorithm in process (d2) will also be performed in very little time. All these factors make the proposed fast MPC method valid and efficient, especially for large-scale structures.

\section{Numerical simulations}

In this section, many numerical simulations will be implemented to verify the validity and efficiency of the proposed fast MPC method. The NS component of the El Centro earthquake in 1940 is chosen as the input external motion in the X-direction for all the cases of the simulations, i.e.

$$
\mathbf{D}_{2} \mathbf{p}=-\mathbf{M E} \ddot{x}_{g}
$$

Where $\mathbf{E}$ is the indicator vector of the excitation with $n \times 1$ dimensions, and $\ddot{x}_{g}$ is the dynamic acceleration of the earthquake. For the El Centro earthquake, the sampling period is $0.02 \mathrm{~s}$. Assuming 
the number of time intervals is 1,000 , and all the numerical simulations are performed on a personal computer with an Intel(R) core(TM) i7-4800MQ (2.70 GHz) processor and 8 GB RAM, running on Windows7 64bit. All algorithms are implemented in the MATLAB R2012a computing environment.

\subsection{Problem description}

Adjacent building is an important and common structure in civil engineering. Adjacent buildings are conjoined together by some linkers and have influences between each other. The basic characteristic of adjacent frame building is that the main structures are established by beams, columns and floors. A plane adjacent frame structure is considered in this paper, as shown in Fig. 2. To capture the important characteristics of adjacent buildings and make the problem manageable, the frame is modeled by two-dimensional beam elements with the same geometry and size. The adjacent buildings are also connected by beam elements, but their density properties are all set to zero. All the bases of the frame are fixed to the ground. To control the plane frame, 10 actuators are placed at each floor on the right side of the frame, and on the other side are the observation points for control. The output variables for control are the states of all junctions of the beams and columns. The geometry and material parameter information of the frame are all given in Table 3. Besides, we mainly choose weighting matrices according to the Bryson's rule, i.e., weighting matrices are taken as diagonal matrices, and the value of the diagonal elements are determined by the maximum acceptable value of output $\mathbf{y}$ and control $\mathbf{u}$. In order to facilitate the algorithm test, the weighting matrices are chosen to be $\mathbf{Q}=1.0 \times 10^{8} \times \mathbf{I}_{3}$, and $\mathbf{R}=0.01 \times \mathbf{I}_{4}$, where $\mathbf{I}_{3}$ and $\mathbf{I}_{4}$ are $N p \times N p$ and $N m \times N m$ unit matrices.

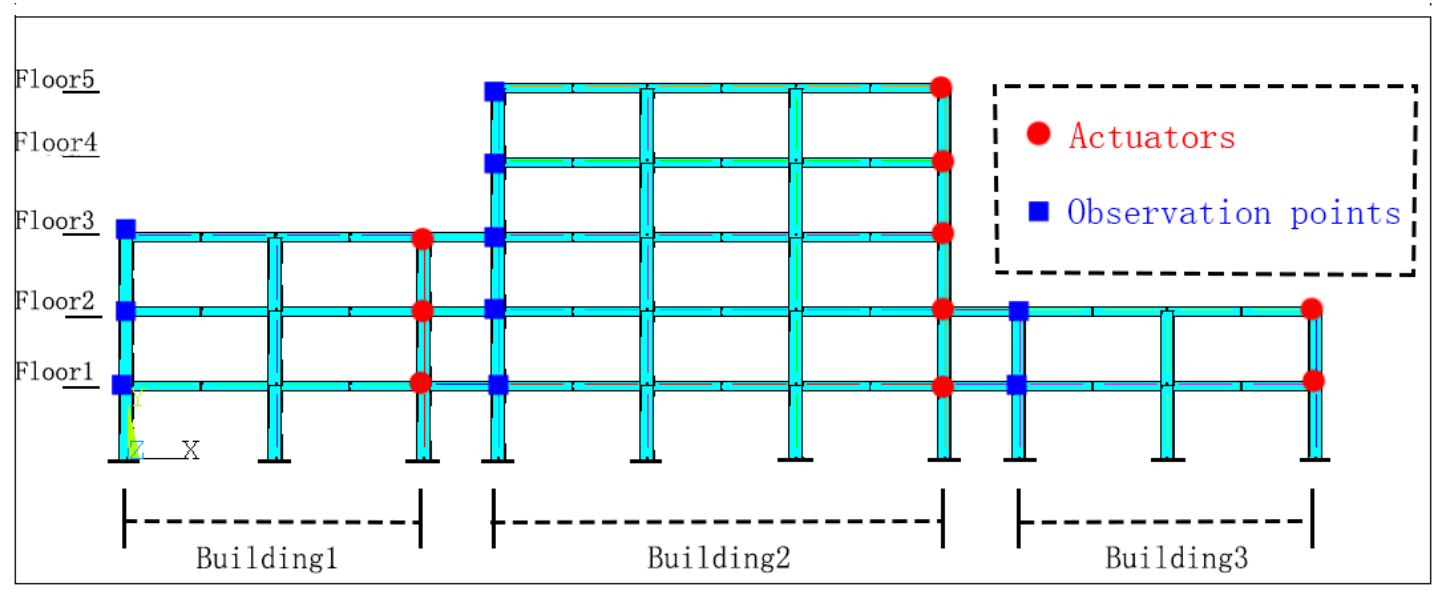

Fig. 2. Structural model of the adjacent buildings 
Table 3 The geometry and material parameters information

\begin{tabular}{ccc}
\hline Beams & Columns & Others \\
\hline Section $\left(\mathrm{m}^{2}\right):$ & Section $\left(\mathrm{m}^{2}\right):$ & Interlayer heights $(\mathrm{m}):$ \\
$0.3 \times 0.5$ & $0.7 \times 0.7$ & 4.0 \\
Poisson's ratio : & Poisson's ratio : & Bay widths (m): \\
0.23 & 0.25 & 8.0 \\
Density $\left(\mathrm{kg} / \mathrm{m}^{3}\right):$ & Density $\left(\mathrm{kg} / \mathrm{m}^{3}\right):$ & Rayleigh-Dumping: \\
1200 & 2500 & $\gamma=0.02, \zeta=0.03$ \\
\hline
\end{tabular}

\subsection{Simulation results by the proposed fast MPC method}

In this sub-section, the element size is set to be $4 \mathrm{~m}$, and the number of degrees of freedom is 360 . The prediction horizon is set to be $0.1 \mathrm{~s}$, and its discrete step length for the prediction horizon is set to be $0.02 \mathrm{~s}$. The simulation time is $20 \mathrm{~s}$, and its sampling step length is set to be $0.02 \mathrm{~s}$, which is the same as the sampling period of the El Centro earthquake. The limit control input is set to be $\pm 4 \mathrm{kN}$ for each actuator. The initial conditions are $\mathbf{q}_{0}=0$ and $\dot{\mathbf{q}}_{0}=0$. The simulation results are presented in Fig. 3(a-d). To prove the correctness of the proposed fast MPC method, the upper and lower limits of the actuators are magnified to be $100 \mathrm{kN}$ and $-100 \mathrm{kN}$, and these amplitudes of the control input are greater than those without input saturation, i.e., the ideal controlled case. As shown in Fig. 4(a-d), we can compare the results of the two situations and show the validity of the proposed fast MPC algorithm. Obviously, if the proposed fast MPC algorithm is valid, the simulation results must be coincident. The simulation results for the uncontrolled, ideal-controlled, and saturation-controlled cases are displayed in Fig. 5(a-b).
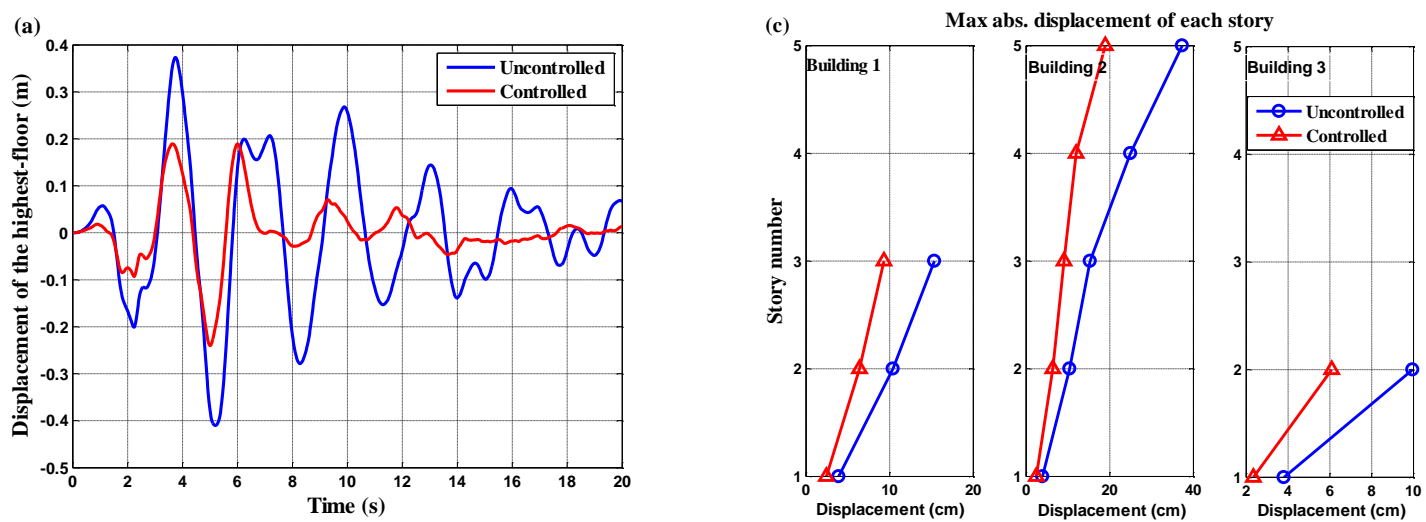

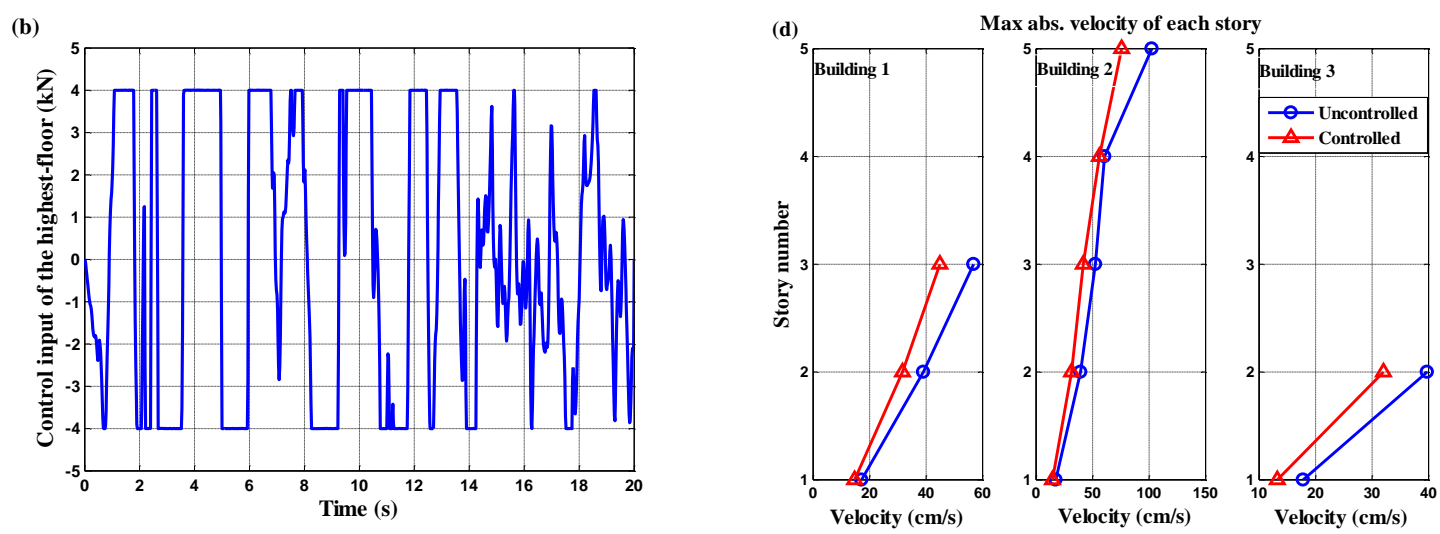

Fig. 3. Control results of saturated-controlled and uncontrolled cases: (a) time history of the displacement of the highest floor of Building 2; (b) time history of the corresponding control input; (c) maximum absolute displacement of each floor; (d) maximum absolute velocity of each floor.
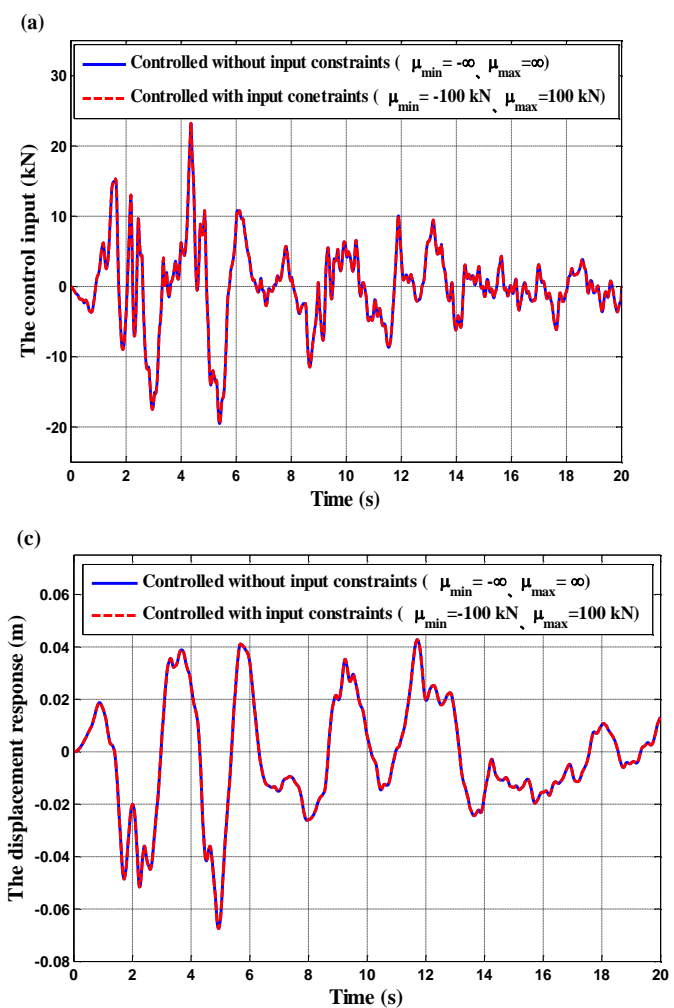
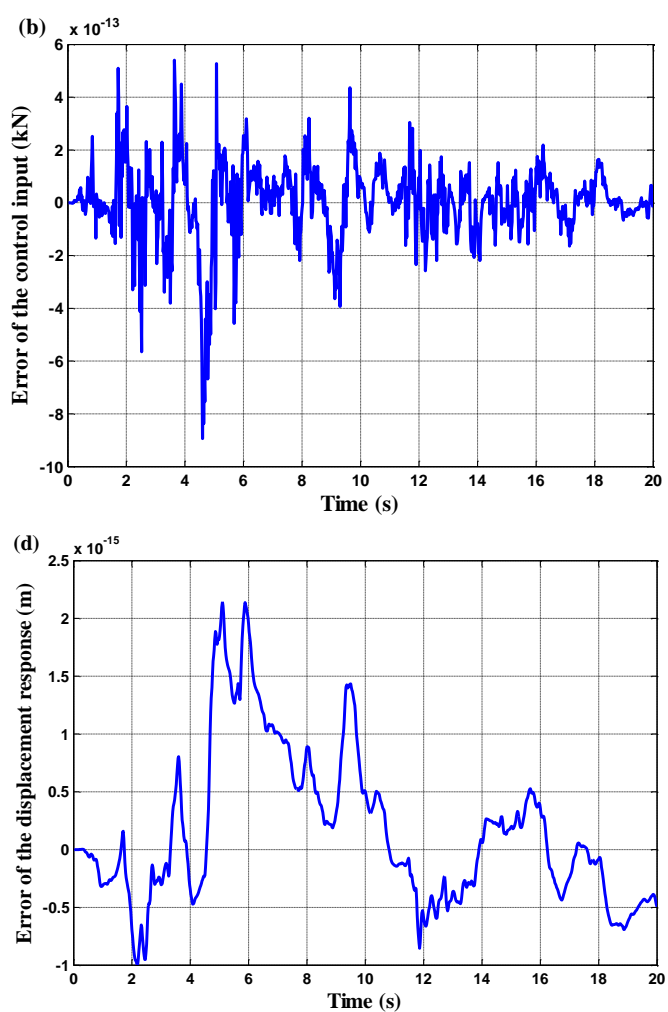

Fig. 4. Control results of the highest floor of Building 2 with/without input constraints: (a) time history of the control input; (b) absolute error of the control input; (c) time history of the displacement response; (d) absolute error of the displacement response 
(a)

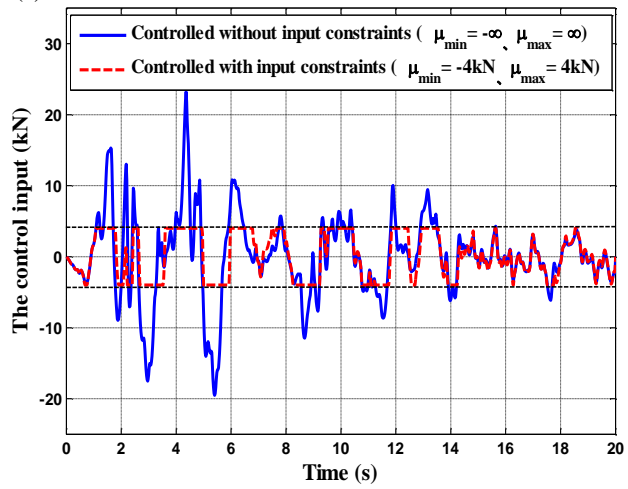

(b)

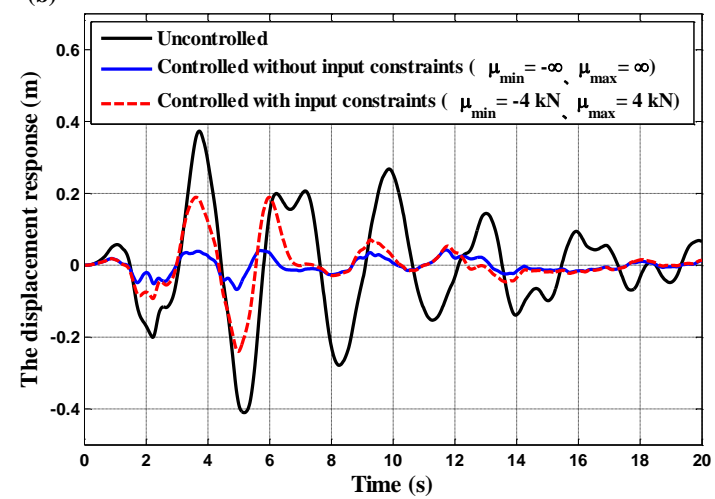

Fig. 5. Simulation results among uncontrolled, ideal-controlled, and saturation-controlled cases: (a) time history of the control input; (b) time history of the displacement response.

As seen from Fig. 3(a), the maximal absolute displacement of the highest floor is reduced from $0.3733 \mathrm{~m}$ to $0.1893 \mathrm{~m}$. The control input of the highest floor is correspondingly constrained between $-4 \mathrm{kN}$ and $4 \mathrm{kN}$, as shown in Fig. 3(b). Furthermore, as shown in Fig. 3(c-d), both the controlled maximum absolute displacement and velocity of each floor are on the left side of those uncontrolled results, which means that the responses have observably decreased. Fig. 4(b) and Fig. 4(d) illustrate that the order of magnitude for the error of the control input is $10^{-13} \mathrm{kN}$, and that for the error of the displacement response is $10^{-15} \mathrm{~m}$. These results clearly show that the proposed fast MPC method with control input constraints can be degenerated into the ideal case without control input constraints.

For further study of the control effect of the proposed fast MPC method, with the change of the upper and lower limits of the input, the reduction of the root-mean-square (RMS) displacement of the highest floor for building 2 is taken as an evaluated performance, i.e.,

$$
\phi=\left(\max _{\mathrm{i}}\left\|q_{\mathrm{i}}(\mathrm{t})\right\|\right) /\left\|q_{\max }\right\|
$$

where $q_{\mathrm{i}}(\mathrm{t})$ is the absolute displacement of the $i$ th floor, and $q_{\max }$ denotes the peak uncontrolled absolute displacement. Then, we change the bounds from $\pm 3 \mathrm{kN}$ to $\pm 30 \mathrm{kN}$, and the control effect will be changed as shown in Fig. 6. 


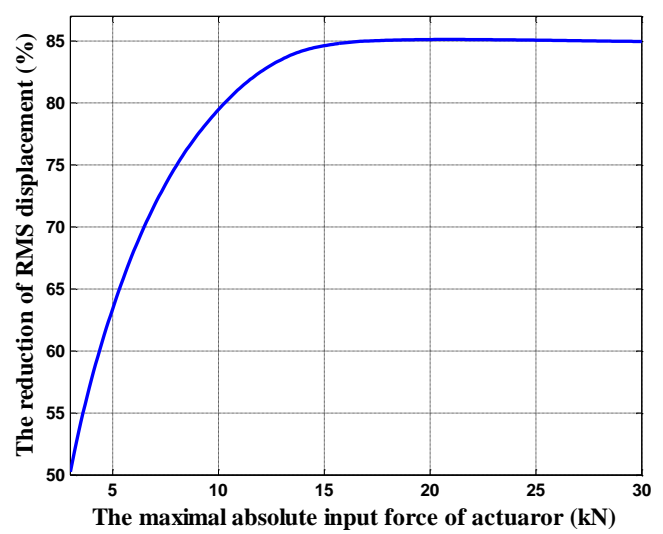

Fig. 6. Reduction of root-mean-square displacement for different max absolute control input.

As seen in Fig. 6, with the increasing of the input constraint bounds $\mu$, the percentage reduction first increases quickly, and then it tends to be stable. As $\mu$ increases from $3 \mathrm{kN}$ to $20 \mathrm{kN}$, the percent reduction increases from $50.53 \%$ to the peak of $85.12 \%$. Then, with the increasing of the input constraint bounds $\mu$, the percent reduction remains stable at approximately $85.11 \%$. Therefore, Fig. 6 can be used as a resource for the specification of the actuators.

In a brief summary, when the input constraints are relaxed enough, the simulation results from the proposed fast MPC method are coincident with those from the ideal case of no input limits. This indicates that the proposed fast MPC with actuator saturation is correct and valid.

\subsection{Accuracy comparison between QPC, Quadprog, and the proposed fast method}

For the comparison between the standard MPC1 and the proposed fast MPC method, the element size is set to be $0.5 \mathrm{~m}$, and the number of degrees of freedom is 3930 . The results obtained with the two method are presented in Fig.7.
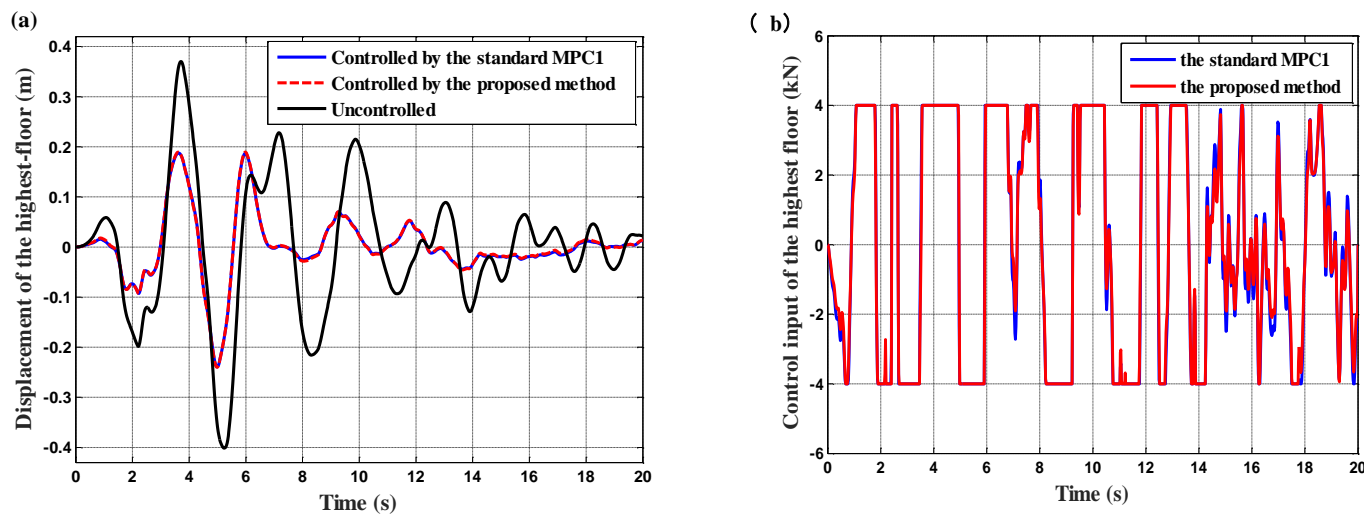

Fig. 7. Control results of the standard MPC1 and the proposed fast MPC: (a) time history of the displacement response; (b) time history of the control input. 
As can be seen from Fig. 7(a), the vibrations of the highest floor are greatly suppressed. Furthermore, the displacement of the proposed fast MPC method is quite consistent with that of the standard MPC1. And a slightly smaller control input is required of the proposed fast MPC method as shown in Fig. 7(b). These results illustrate that both the above two methods can effectively control the vibration of the structure, and the proposed fast MPC method has the same computation precision as the standard MPC1.

For the comparison between the standard MPC2 and the proposed fast MPC method, first, a small-scale structure system with 360 degrees of freedom is used for analysis. The other parameters are selected that the length of the predictive step is $\mathrm{Dt}=0.02 \mathrm{~s}$ and the sampling period is $\mathrm{dt}=0.02 \mathrm{~s}$. Numerical simulations for this case shown that the standard MPC2 are divergent and the proposed fast MPC method is carried out efficiently. Only when the length of the predictive step is reduced to $1 / 20$, i.e., $\eta=\mathrm{Dt} / 20$ and the sampling period is not changed can the standard MPC2 be used normally. With further reductions, the simulation results of the standard MPC2 will gradually converge to that computed by the proposed fast MPC method. Fig. 8 shows the time history of the displacement of the highest floor under different lengths of predictive step. As seen, both the standard MPC2 and the proposed fast MPC method can suppress the vibration of the structure. When the length of predictive step is decreased to $1 / 20,1 / 23,1 / 26$ or $1 / 29$, the curves obtained by the standard MPC2 gradually tend to the curve obtained by the proposed fast MPC method. These simulation results demonstrate that the proposed fast MPC method is effective and can be applied with a large length of predictive step.

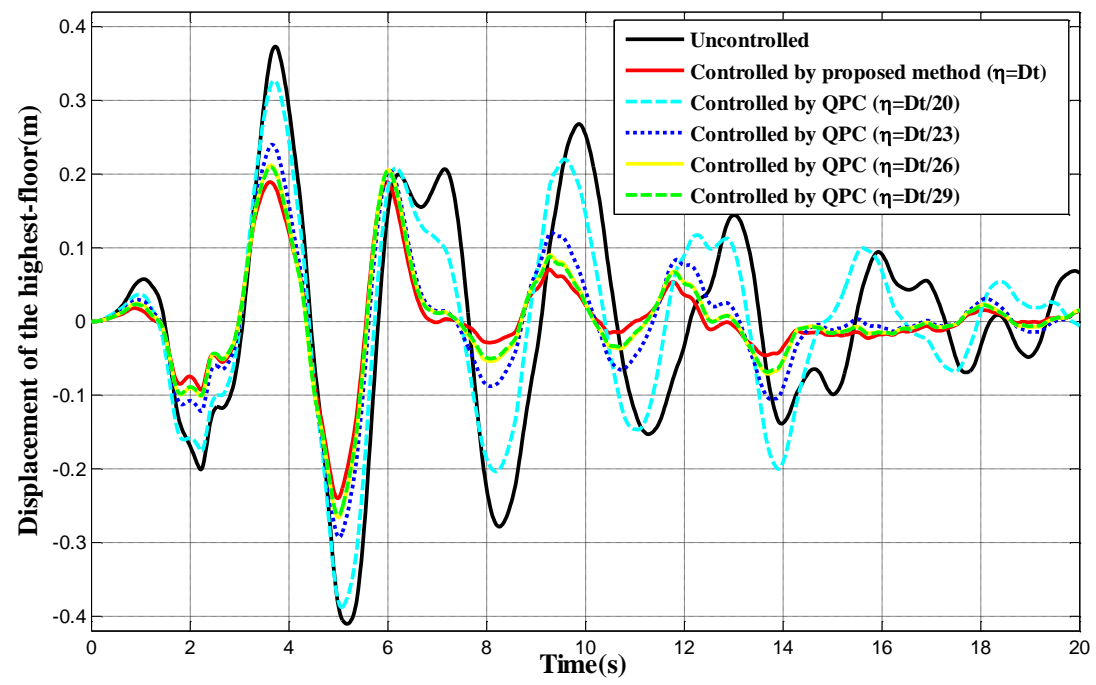

Fig. 8. Time history of the displacement with different lengths of predictive step 


$$
\pi
$$


Second, the element size of the plane adjacent frame is set to be $1 \mathrm{~m}$, and resulting in a model with 1890 degrees of freedom. For this larger-scale case, when only the length of the predictive step is reduced and the sampling period is not changed, the standard MPC2 will be divergent. Therefore, the linear interpolation is applied for the El Centro earthquake and to obtain the new excitation with a smaller sampling period. For a quantitative description, let the length of predictive step Dt $=0.02 / 1 \mathrm{~s}$, $0.02 / 100 \mathrm{~s}, 0.02 / 200 \mathrm{~s}, \ldots, 0.02 / 500 \mathrm{~s}$, and the sampling period $\mathrm{dt}=0.02 / 1 \mathrm{~s}, 0.02 / 10 \mathrm{~s}, 0.02 / 20 \mathrm{~s}, \ldots$, 0.02/100 s. The different control effects of the standard MPC2 solved by QPC or Quadprog are listed in Table 4. For all the above parameters of Dt and dt, the proposed fast MPC method can give the right convergence results, and these results are not included in Table 4. However, as seen from Table 4, when Dt and dt are both reduced by 100 times, the standard MPC2 will be not divergent and will control effectively.

For further study on the control system's quantitative performance, two evaluation indexes are used. The first evaluation index is a measure of the reduction in the peak interstory drift, given as

$$
\boldsymbol{J}_{1}=\left(\max _{\mathrm{t}, \mathrm{i}} \frac{\left|\boldsymbol{d}_{\mathrm{i}}(\mathrm{t})\right|}{h_{\mathrm{i}}}\right) /\left(\max _{\mathrm{t}, \mathrm{i}} \frac{\left|\boldsymbol{d}_{\mathrm{i}}^{0}(\mathrm{t})\right|}{h_{\mathrm{i}}}\right)
$$

The second evaluation index is a measure of the RMS interstory drift, given as

$$
\boldsymbol{J}_{2}=\left(\max _{\mathrm{i}} \frac{\left\|\boldsymbol{d}_{\mathrm{i}}(\mathrm{t})\right\|}{h_{\mathrm{i}}}\right) /\left(\max _{\mathrm{i}} \frac{\left\|\boldsymbol{d}_{\mathrm{i}}^{0}(\mathrm{t})\right\|}{h_{\mathrm{i}}}\right)
$$

where $h_{\mathrm{i}}, \boldsymbol{d}_{\mathrm{i}}(\mathrm{t})$ and $\boldsymbol{d}_{\mathrm{i}}^{0}(\mathrm{t})$ are the interstory height and the interstory drifts of the aboveground floors over the response history and in uncontrolled response.

Now, the control performance of each method can be checked. Let the length of the predictive step $\mathrm{Dt}=0.02 / 100,0.02 / 120,0.02 / 140, \ldots, 0.02 / 500$, the sampling period $\mathrm{dt}=0.02 / 100 \mathrm{~s}$, and the simulation time be $20 \mathrm{~s}$. Then, the simulation results of the standard MPC2 are substituted into Eq.(50) and Eq.(51) to obtain the evaluation index. Fig. 9 shows the change in the evaluation index under different lengths of predictive step. 


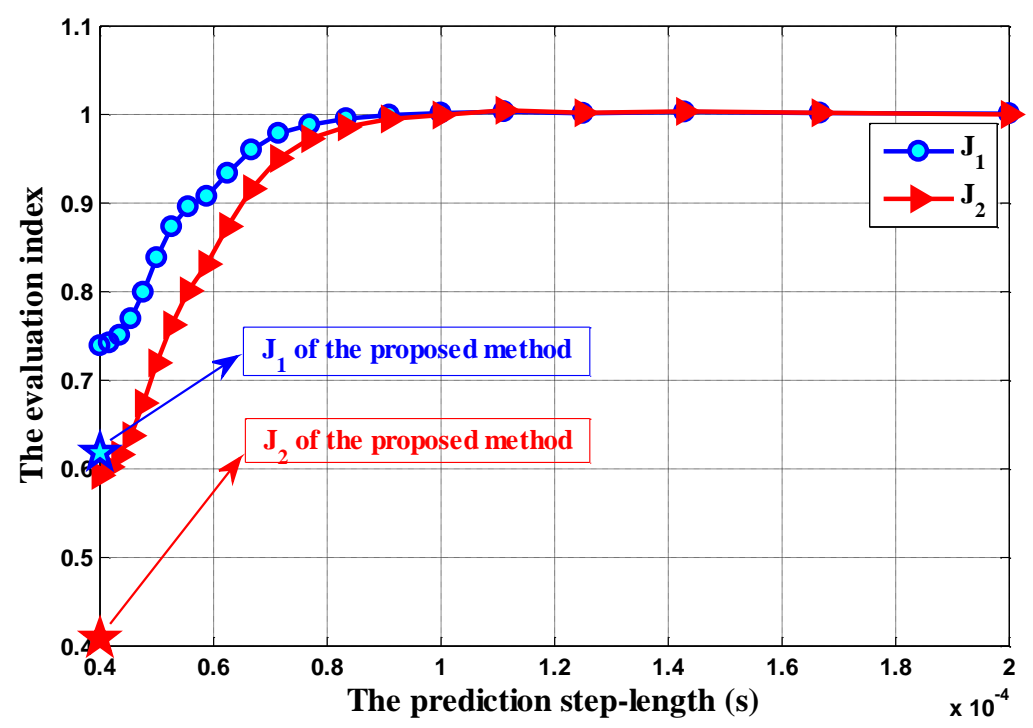

Fig.9. The influence of different predictive step-lengths for evaluation index

As seen from right to the left in Fig. 9, when the length of predictive step is reduced from 100 times $\left(\mathrm{Dt}=2 \times 10^{-4}\right.$ s) to 200 times $\left(\mathrm{Dt}=1 \times 10^{-4} \mathrm{~s}\right)$, both the evaluation indexes $\mathrm{J}_{1}$ and $\mathrm{J}_{2}$ tend to 1 . These results mean that there is no significant control effect. Furthermore, with the decreasing of the length of the predictive step, the evaluation indexes gradually go down. This means that the control performance of the standard MPC2 is becoming better. When the length of the predictive step is reduced to $1 / 500\left(\mathrm{Dt}=4 \times 10^{-5} \mathrm{~s}\right)$, the evaluation indexes fall to their lowest points, i.e., $\mathrm{J}_{1}=0.7392$ and $\mathrm{J}_{2}=0.5926$. However, for the proposed fast MPC method, i.e., the marks of the star in Fig. 9, even under large lengths of the predictive step and sampling period, the evaluation indexes are $\overline{\mathbf{J}}_{1}=0.6183$ and $\overline{\mathbf{J}}_{2}=0.4085$. Compared with those from the standard MPC2, they are reduced by $16.36 \%$ and $31.07 \%$, respectively.

In a brief summary, the preceding analysis of the two different scales of structure system by the standard MPC2 and the proposed fast MPC in this sub-section shows one of the advantages of the proposed fast MPC method, that it can be applied under a large length of predictive step and be stable for a long time. In addition, the proposed fast MPC method has the same computation precision as the standard MPC1. However, the standard MPC2 can only be carried out under a smaller length of predictive step. Compared with the proposed method, the smaller the step-length, the closer the displacement responses and the evaluation indexes are. 


\subsection{Efficiency comparison between QPC, Quadprog, and the proposed fast method}

To demonstrate the high computing efficiency of the proposed method for different scales of structure, the element size of the plane adjacent frame is set to $4 \mathrm{~m}, 2 \mathrm{~m}, 1 \mathrm{~m}, 0.5 \mathrm{~m}, 0.25 \mathrm{~m}$ and $0.125 \mathrm{~m}$, with the number of degrees of freedom $n=360,870,1890,3930,8010$ and 16170, respectively. The El Centro earthquake has been considered as the external force, and the other conditions are the same as the previous sub-sections. The comparisons of the maximum memory requirements, on-line CPU time and evaluation indexes are listed in Table 5. The length of the predictive step and the sampling period are set to $\mathrm{Dt}=0.02 \mathrm{~s}$ and $\mathrm{dt}=0.02 \mathrm{~s}$ for the standard MPC1 method and the proposed fast MPC method. For the standard MPC2 method, the length of the predictive step is reduced to $1 / 30(\eta=\mathrm{Dt} / 30)$ and the sampling period is not changed for the small-scale structure with 360 degrees of freedom, while for the larger-scale structures, the length of the predictive step and the sampling period are reduced to $1 / 100$ $(\eta=\mathrm{Dt} / 100)$ and $1 / 500\left(\eta^{\prime}=\mathrm{dt} / 500\right)$, respectively. In addition, the matrix exponential in standard MPC1 is computed by using the expm function in MATLAB, which is based on the Padé approximation with scaling and squaring. The sparse matrix format is used in all methods, and the simulation results are also obtained by the sparse solvers in MATLAB.

Table 5 Comparisons of the memory requirement, on-line CPU time and evaluation index

\begin{tabular}{|c|c|c|c|c|c|c|c|}
\hline \multirow{2}{*}{ Performances } & \multirow{2}{*}{ Methods } & \multicolumn{5}{|c|}{ Degree of freedom $n$} & \multirow[b]{2}{*}{16170} \\
\hline & & 360 & 870 & 1890 & 3930 & 8010 & \\
\hline \multirow{5}{*}{$\begin{array}{c}\text { Memory } \\
\text { Requirement } \\
\text { (G) }\end{array}$} & Proposed Method & 0.0143 & 0.0248 & 0.0861 & 0.1806 & 0.3754 & 0.4137 \\
\hline & MPC1 (QPC) & 0.0330 & 0.2700 & 1.2203 & 5.1287 & - & - \\
\hline & MPC1 (Quadprog) & 0.0303 & 0.2015 & 1.2265 & 5.0633 & - & - \\
\hline & MPC2 (QPC) & 0.0565 & 1.9300 & 5.5485 & - & - & - \\
\hline & MPC2 (Quadprog) & 0.0239 & 0.9898 & 4.2653 & 7.8691 & - & - \\
\hline \multirow{5}{*}{$\begin{array}{c}\text { On-line CPU } \\
\text { Time } \\
\left(10^{-3} \mathrm{~s}\right)\end{array}$} & Proposed Method & 2.2606 & 2.4225 & 2.9063 & 3.6574 & 5.5465 & 11.6364 \\
\hline & MPC1 (QPC) & 0.2526 & 0.3065 & 0.5091 & 1.1215 & - & - \\
\hline & MPC1 (Quadprog) & 6.0714 & 6.1510 & 6.4587 & 7.0288 & - & - \\
\hline & MPC2 (QPC) & 3.0049 & 4.4001 & 4.3025 & - & - & - \\
\hline & MPC2 (Quadprog) & 6.1700 & 52.6000 & 57.6000 & 61.0500 & - & - \\
\hline \multirow{6}{*}{$\begin{array}{l}\text { Evaluation } \\
\text { Index } \\
\text { J1 }\end{array}$} & Proposed Method & 0.6192 & 0.6407 & 0.6183 & 0.5759 & 0.5782 & 0.5853 \\
\hline & MPC1 (QPC) & 0.6157 & 0.6364 & 0.6149 & 0.5732 & - & - \\
\hline & MPC1 (Quadprog) & 0.6157 & 0.6364 & 0.6149 & 0.5732 & - & - \\
\hline & MPC2 (QPC) & 0.6542 & 0.7387 & 0.7392 & - & - & - \\
\hline & MPC2 (Quadprog) & 0.6542 & 0.7387 & 0.7392 & 0.8409 & - & - \\
\hline & Reduction (\%) & 5.35 & 13.27 & 16.36 & 31.51 & - & - \\
\hline Evaluation & Proposed Method & 0.4806 & 0.3975 & 0.4085 & 0.5759 & 0.5533 & 0.5534 \\
\hline & & & 27 & & & & \\
\hline
\end{tabular}




\begin{tabular}{cccccccc}
\hline Index & MPC1 (QPC) & 0.4798 & 0.3964 & 0.4076 & 0.5204 & - & - \\
J2 & MPC1 (Quadprog) & 0.4798 & 0.3964 & 0.4076 & 0.5204 & - & - \\
& MPC2 (QPC) & 0.5042 & 0.5588 & 0.5926 & - & - & - \\
& MPC2 (Quadprog) & 0.5042 & 0.5588 & 0.5926 & 0.8282 & - & - \\
& Reduction (\%) & 4.68 & 28.87 & 31.07 & 30.46 & - & - \\
\hline
\end{tabular}

Note: The CPU time is the CPU time for one sampling period; The Reduction is the results between the standard MPC2 method and the proposed fast MPC method.

For the comparisons between the proposed fast MPC method and the standard MPC1 method: As can be seen from the Table 5, the maximum memory of the standard MPC1 is larger than that of the proposed fast MPC method, especially for the large-scale structure. When $n=8010$, the memory requirement even exceeds the maximum memory of the personal computer ( $8 \mathrm{G}$ is available), while for the proposed fast MPC method, the memory requirement is only $0.3754 \mathrm{G}$. It can also be observed from the table that the on-line CPU time for one sampling period of the proposed fast MPC method is longer than that of the standard MPC1, which is consistent with the analysis in the preceding section 4. However, even $n$ increases to 16170 , the on-line time is only 0.0116 s, which is shorter than the sampling period 0.02s. Besides, the evaluation index including $\mathrm{J}_{1}$ and $\mathrm{J}_{2}$ of the standard MPC1 are both nearly the same with those of the proposed fast MPC method.

For the comparisons between the proposed fast MPC method and the standard MPC2 method: In Table 5, the maximum memory requirement, the on-line CPU time and the evaluation indexes of the standard MPC2 are all larger than those of the proposed fast MPC method. First, when the degrees of freedom are $n=3930$, the memory requirement of the standard MPC2 solved by QPC has exceeded the maximum memory of the personal computer, and that solved by Quadprog reaches 7.8691 G. While for the proposed fast MPC method, when the degrees of freedom are $n=8010$, the maximum memory requirement is only 0.3754 G. Second, the standard MPC2 solved by Quadprog is very memory-consuming and time-consuming. When the degrees of freedom are $n=1890$, the CPU time for one sampling period is $57.60 \times 10^{-3} \mathrm{~s}$, while for the proposed fast MPC method, the CPU time is just $2.91 \times 10^{-3} \mathrm{~s}$. Moreover, comparing with the standard MPC2, the evaluation indexes of the proposed fast MPC method are much smaller for all numbers of degrees of freedom. For the small-scale structure ( $n=360$ ), the evaluation indexes $\mathrm{J}_{1}$ and $\mathrm{J}_{2}$ are reduced by $5.35 \%$ and $4.68 \%$, respectively. With the increasing number of degrees of the freedom, the difference will be more obvious. For example, when the degrees of freedom are $n=1890$, the evaluation indexes $\mathrm{J}_{1}$ and $\mathrm{J}_{2}$ are reduced by $16.36 \%$ and 
$31.07 \%$, respectively. That is to say, the control performance of the proposed fast MPC method is better than that of the standard MPC2.

Based on the preceding results in this sub-section, another advantage of the proposed fast MPC method is that it is still very memory-saving and has high computing efficiency on the premise of favorable control performance.

\section{Conclusions}

In this paper, a fast and accurate model predictive control with actuator saturation is presented for large-scale structures. Based on the second-order dynamic equation, the explicit expression form of the Newmark- $\beta$ method is derived. Then, based on the parametric variational principle, the model predictive control problem with input saturation is transformed into a linear complementarity problem. The optimal control input can be achieved by one linear complementarity problem and one transient analysis problem. To improve the computing efficiency and save memory, the physical meaning of the explicit expression form of the Newmark- $\beta$ method is sufficiently explained and applied for fast computation. The numerical simulations indicate that (1) The proposed fast MPC method is an accurate and valid method, and the proposed fast MPC method with a control input constraint can be degenerated into the ideal case without a control input constraint; (2) The proposed fast MPC method is highly efficient and very memory-saving, especially for large-scale structural dynamic control problems; and (3) The proposed fast MPC method can give satisfactory control results for a long time under large lengths of predictive step and sampling period.

\section{Acknowledgements}

The authors are grateful for the financial support of the National Science Foundation of China (11472069); the Fundamental Research Funds for the Central Universities (DUT16LK21); and the Dalian Science and Technology Project (2015R048, 2015A11GX037).

\section{Appendix-A}

"The prediction horizon" refers to the length of future output vector, and the number of the expected output values in the future after time $t_{k}$. "The input horizon" refers to the length of future input vector, and the number of the control input values in the future after time $t_{k}$. Therefore, In practical implementations, the prediction horizon (that is defined as $N$ in Eq. (12)) can be larger than 
the input horizon, so we can define the input horizon as $S(1 \leq S \leq N)$, and consider that $\boldsymbol{u}_{S-1}=\boldsymbol{u}_{S}=\boldsymbol{u}_{S+1}=\cdots=\boldsymbol{u}_{N-1}$. Then we can establish a conversion relation $\mathbf{U}=\mathbf{L} \overline{\mathbf{U}}$, given as

$$
\mathbf{U}=\left\{\begin{array}{c}
\mathbf{u}_{0} \\
\mathbf{u}_{1} \\
\vdots \\
\mathbf{u}_{S-1} \\
\mathbf{u}_{S-1} \\
\vdots \\
\mathbf{u}_{S-1}
\end{array}\right\}=\left[\begin{array}{cccc}
\mathbf{I}_{\mathrm{m}} & \mathbf{0} & \mathbf{0} & \mathbf{0} \\
\mathbf{0} & \mathbf{I}_{\mathrm{m}} & \mathbf{0} & \mathbf{0} \\
\vdots & \vdots & \ddots & \\
\mathbf{0} & \mathbf{0} & \mathbf{0} & \mathbf{I}_{\mathrm{m}} \\
\mathbf{0} & \mathbf{0} & \mathbf{0} & \mathbf{I}_{\mathrm{m}} \\
\vdots & \vdots & \vdots & \vdots \\
\mathbf{0} & \mathbf{0} & \mathbf{0} & \mathbf{I}_{\mathrm{m}}
\end{array}\right]\left\{\begin{array}{c}
\mathbf{u}_{0} \\
\mathbf{u}_{1} \\
\vdots \\
\mathbf{u}_{S-1}
\end{array}\right\}=\mathbf{L} \overline{\mathbf{U}}
$$

Substituting the above Eq.(A1) into Eq. (12), the future outputs can be obtained as

$$
\mathbf{Y}=\overline{\mathbf{F}} \mathbf{x}_{0}+\overline{\mathbf{G}}(\mathbf{L} \overline{\mathbf{U}})
$$

Then making a similar operation as Eq.(13), Eq.(15) and Eq.(16), the QP problem can be modified as

$$
\left\{\begin{array}{l}
\min _{\overline{\mathbf{U}}} \frac{1}{2} \overline{\mathbf{U}}^{\mathrm{T}}\left(\mathbf{L}^{\mathrm{T}} \overline{\mathbf{G}}^{\mathrm{T}} \mathbf{Q} \overline{\mathbf{G}} \mathbf{L}+\mathbf{L}^{\mathrm{T}} \mathbf{R} \mathbf{L}\right) \overline{\mathbf{U}}+\left(\mathbf{L}^{\mathrm{T}} \overline{\mathbf{G}}^{\mathrm{T}} \mathbf{Q} \overline{\mathbf{F}} \mathbf{x}_{0}\right)^{\mathrm{T}} \overline{\mathbf{U}} \\
\text { s.t. } \hat{\mathbf{E}} \boldsymbol{\mu}_{\min } \leq \overline{\mathbf{U}} \leq \hat{\mathbf{E}} \boldsymbol{\mu}_{\max }
\end{array}\right.
$$

Where $\hat{\mathbf{E}}$ is an $m S \times m$ matrix for enlarged dimensions like $\overline{\mathbf{E}}$.

In this paper, we consider that the input horizon is the same as the prediction horizon.

\section{Appendix-B}

The expressions for $\Psi_{\mu v}(\mu, v=1,2,3)$ and $\mathrm{b}_{\kappa}(\kappa=1,2,3,4,5,6)$ in Eq.(22)-(24) are given by

$$
\begin{gathered}
\left\{\begin{array}{l}
\Psi_{11}=\mathrm{b}_{4} \hat{\mathbf{K}}^{-1} \mathbf{M}+\mathrm{b}_{1} \hat{\mathbf{K}}^{-1} \mathbf{C} \\
\Psi_{12}=-\left(\mathrm{b}_{5} \hat{\mathbf{K}}^{-1} \mathbf{M}+\mathrm{b}_{2} \hat{\mathbf{K}}^{-1} \mathbf{C}\right) \\
\Psi_{13}=-\left(\mathrm{b}_{6} \hat{\mathbf{K}}^{-1} \mathbf{M}+\mathrm{b}_{3} \hat{\mathbf{K}}^{-1} \mathbf{C}\right)
\end{array}\right. \\
\left\{\begin{array}{l}
\Psi_{21}=\mathrm{b}_{1} \mathrm{~b}_{4} \hat{\mathbf{K}}^{-1} \mathbf{M}+\mathrm{b}_{1} \mathrm{~b}_{1} \hat{\mathbf{K}}^{-1} \mathbf{C}-\mathrm{b}_{1} \mathbf{I}_{1} \\
\Psi_{22}=-\left(\mathrm{b}_{1} \mathrm{~b}_{5} \hat{\mathbf{K}}^{-1} \mathbf{M}+\mathrm{b}_{1} \mathrm{~b}_{2} \hat{\mathbf{K}}^{-1} \mathbf{C}-\mathrm{b}_{2} \mathbf{I}_{1}\right) \\
\Psi_{23}=-\left(\mathrm{b}_{1} \mathrm{~b}_{6} \hat{\mathbf{K}}^{-1} \mathbf{M}+\mathrm{b}_{1} \mathrm{~b}_{3} \hat{\mathbf{K}}^{-1} \mathbf{C}-\mathrm{b}_{3} \mathbf{I}_{1}\right)
\end{array}\right. \\
\left\{\begin{array}{l}
\Psi_{31}=\mathrm{b}_{4} \mathrm{~b}_{4} \hat{\mathbf{K}}^{-1} \mathbf{M}+\mathrm{b}_{1} \mathrm{~b}_{4} \hat{\mathbf{K}}^{-1} \mathbf{C}-\mathrm{b}_{4} \mathbf{I}_{1} \\
\Psi_{32}=-\left(\mathrm{b}_{4} \mathrm{~b}_{5} \hat{\mathbf{K}}^{-1} \mathbf{M}+\mathrm{b}_{2} \mathrm{~b}_{4} \hat{\mathbf{K}}^{-1} \mathbf{C}-\mathrm{b}_{5} \mathbf{I}_{1}\right) \\
\Psi_{33}=-\left(\mathrm{b}_{4} \mathrm{~b}_{6} \hat{\mathbf{K}}^{-1} \mathbf{M}+\mathrm{b}_{3} \mathrm{~b}_{4} \hat{\mathbf{K}}^{-1} \mathbf{C}-\mathrm{b}_{6} \mathbf{I}_{1}\right) \\
\mathrm{b}_{1}=\frac{\delta}{\alpha \Delta \mathrm{t}} ; \mathrm{b}_{2}=1-\frac{\delta}{\alpha} ; \mathrm{b}_{3}=\left(1-\frac{\delta}{2 \alpha}\right) \Delta \mathrm{t} ; \mathrm{b}_{4}=\frac{1}{\alpha(\Delta \mathrm{t})^{2}} ; \mathrm{b}_{5}=\frac{-1}{\alpha \Delta \mathrm{t}} ; \mathrm{b}_{6}=1-\frac{1}{2 \alpha}
\end{array}\right.
\end{gathered}
$$




\section{References}

[1]. Korkmaz S. A review of active structural control: challenges for engineering informatics. Comput. Struct. 2011;89 (23-24):2113-2132.

[2]. Takács G, Rohal'-Ilkiv B. Model Predictive Vibration Control: Efficient Constrained MPC Vibration Control for Lightly Damped Mechanical Structures. UK: Springer London Heidelberg New York Dordrecht; 2012.

[3]. Thenozhi S, Yu W. Advances in modeling and vibration control of building structures. Annu. Rev. Control 2013;37 (2):346-364.

[4]. Takács G. Model predictive control algorithms for active vibration control: a study on timing, performance and implementation properties. J. Vib. Control 2014;20(13): 2061-2080.

[5]. Chen YZ, Zhang S, Peng HJ, Chen BS and Zhang HW. A novel fast model predictive control for large-scale structures. J. Vib. Control 2015; doi:10.1177/1077546315610033.

[6]. Suhariyono A, Goo NS, Park HC. Use of lightweight piezo-composite actuators to suppress the free vibration of an aluminum beam. J. Intell. Mater. Syst. Struct. 2008;19 (1):101-112.

[7]. Mahmoodi SN, Ahmadian M. Active vibration control with modified positive position feedback, J. Dyn. Syst. Meas. Control 2009;131 (4):442-447.

[8]. Narayanan S, Balamurugan V. Finite element modelling of piezolaminated smart structures for active vibration control with distributed sensors and actuators. J. Sound. Vibr. 2003;262 (3):529-562.

[9]. Schulz SL, Gomes HM, Awruch AM. Optimal discrete piezoelectric patch allocation on composite structures for vibration control based on GA and modal LQR. Comput. Struct. 2013;128:101-115.

[10]. Jung UJ, Park GJ. A new method for simultaneous optimum design of structural and control systems. Comput. Struct. 2015;160:90-99.

[11]. Chase JG, Breneman SE, Smith HA. Robust $\mathrm{H} \infty$ static output feedback control with actuator saturation. J. Eng. Mech. 1999;125 (2):225-233.

[12]. Peng HJ, Wu ZG, Zhong WX. Hœ norm computation of linear continuous-time periodic systems by a structure-preserving algorithm. Int. J. Control. 2014;87:131-142.

[13]. Lee SH, Min KW, Lee YC, Chung L. Improved design of sliding mode control for civil structures with saturation problem. Earthq. Eng. Struct. Dyn. 2004;33(11):1147-1164.

[14]. Hu Q. Sliding mode attitude control with L2-gain performance and vibration reduction of flexible spacecraft with actuator dynamics. Acta Astronaut. 2010;67 (5):572-583.

[15]. Valoor MT, Chandrashekhara K, Agarwal S. Self-adaptive vibration control of smart composite beams using recurrent neural architecture. Int. J. Solids Struct. 2001;38 (44-45):7857-7874.

[16]. Kumar R, Mishru B, Jain S. Vibration control of smart composite laminated spherical shell using neural network. J. Intell. Mater. Syst. Struct. 2008;19(8):947-957.

[17]. Du H, Zhang N, Naghdy F. Actuator saturation control of uncertain structures with input time delay. J. Sound Vibr. 2011;330(18-19):4399-4412.

[18]. Kim JH, Jabbari F. Scheduled controllers for buildings under seismic excitation with limited actuator capacity. J. Eng. Mech. 2004;130(7):800-808.

[19]. Venanzi I, Ierimonti L, Ubertini F. An enhanced nonlinear damping approach accounting for system constraints in active mass dampers. J. Sound. Vibr. 2015;357(24):2-15.

[20]. Du H, Zhang H. Energy-to-peak control of seismic-excited buildings with input delay. Struct. Control. Health Monit. 2007;14 (7):947-970.

[21]. Zhao Y, Sun W, Gao H. Robust control synthesis for seat suspension systems with actuator saturation and time-varying input delay. J. Sound Vibr. 2010;329(21):4335-4353.

[22]. Ding YC, Weng FL, Yu ZA. Actuator saturation and control design for buildings structural systems with improved uncertainty description. Shock Vib. 2013;20(2):297-308.

[23]. Morales AL, Rongong JA, Sims ND. A finite element method for active vibration control of uncertain structures. Mech. Syst. Signal Proc. 2012;32:79-93.

[24]. Lim CW. Remarks on robust stability of saturation controllers. J. Sound Vibr. 2007;299(1-2):363-372.

[25]. Weng F, Ding Y, Ge J, Liang L, Yang G. Finite-Time Vibration Control of Earthquake Excited Linear Structures with Input Time-Delay and Saturation. J. Low Freq. Noise Vib. Act. Control 2014; 33 (3):245-272.

[26]. Materazzi AL, Ubertini F. Robust structural control with system constraints. Struct. Control. Health. Monit. 2012;19(3):472-490.

[27]. Nagashima I, Shinozaki Y. Variable gain feedback control technique of active mass damper and its 
application to hybrid structural control. Earthq. Eng. Struct. Dyn. 1997;26 (8):815-838.

[28]. Dìaz I, Reynolds P. On-off nonlinear active control of floor vibrations. Mech. Syst. Signal Proc. 2010;24 (6):1711-1726.

[29]. Mehrpouya MA, Fallahi S. A modified control parameterization method for numerical solution of bang-bang optimal control problems. J. Vib. Control 2015;21(12):2407-2415.

[30]. Cychowski M, Szabat K, Orlowska-Kowalska T. Constrained model predictive control of the drive system with mechanical elasticity. IEEE Trans. Ind. Electron. 2009;56(6):1963-1973.

[31]. Du H, Zhang N, Samali B, Naghdy F. Robust sampled-data control of structures subject to parameter uncertainties and actuator saturation. Eng. Struct. 2012;36:39-48.

[32]. Peng HJ, Gao Q, Wu ZG, Zhong WX. Fast model predictive control method for large-scale structural dynamical systems. Shock Vib. 2014;Article ID 508954:1-13.

[33]. Fischer A, Eberhard P. Controlling vibrations of a cutting process using predictive control. Comput. Mech. 2014;54(1):21-31.

[34]. Peng HJ, Gao Q, Wu ZG, Zhong WX. Optimal guidance based on receding horizon control for low-thrust transfer to libration point orbits. Adv. Space Res. 2013;51(11):2093-2111.

[35]. Wills AG, Bates D, Fleming AJ, Ninness B, Moheimani SQR. Model predictive control applied to constraint handling in active noise and vibration control. IEEE Trans. Control Syst. Technol. 2008;16 (1):3-12.

[36]. Kvasnica M, Löfberg J, Fikar M. Stabilizing polynomial approximation of explicit MPC. Automatica 2011;47(10):2292-2297.

[37]. Leomanni M, Rogers E, Gabriel SB. Explicit model predictive control approach for low-thrust spacecraft proximity operations. J. Guid. Control Dyn. 2014;37(6): 1780-1790.

[38]. Takács G, Rohal'-Ilkiv B. Newton-Raphson based efficient model predictive control applied on active vibrating structures. In: Proceedings of the European control conference, Budapest; 2009.

[39]. Kouvaritakis B, Li S, Cannon M. A line search improvement of efficient MPC. Automatica 2010;46(11):1920-1924.

[40]. Gould N, Toint PL. Preprocessing for quadratic programming. Math. Program. Ser. B 2004;100 (1):95-132.

[41]. Coleman TF, Li Y. A reflective newton method for minimizing a quadratic function subject to bounds on some of the variables. SIAM J. Optim. 1996; 6(4):1040-1058.

[42]. Gill PE, Murray W, Wright MH. Practical Optimization. UK: Academic Press, London; 1981.

[43]. Wills A, Knagge G, Ninness B. Fast linear model predictive control via custom integrated circuit architecture. IEEE Trans. Control Syst. Technol. 2012;20(1):59-71.

[44]. Clough RW, Penzien J. Dynamics of Structures. USA:McGraw-Hill Companies, New York; 1975.

[45]. Peng HJ, Gao Q, Zong WX, et al. Parametric variational solution of linear-quadratic optimal control problems with control inequality constraints. Appl. Math. Mech.-Engl. Ed. 2014;35(9):1079-1098.

[46]. Wright SJ. Primal-Dual Interior-Point Methods. USA: Society for Industrial and Applied Mathematics, Philadelphia; 1997.

[47]. Billups AC, Murty KC. Complementarity problems. J. Comput. Appl. Math. 2000;124:303-318. 